\title{
Cue Effects on Memory for Location When Navigating Spatial Displays
}

\author{
Sylvia Fitting, Douglas H. Wedell, Gary L. Allen \\ Department of Psychology, University of South Carolina
}

Received 28 May 2008; received in revised form 21 May 2009; accepted 8 June 2009

\begin{abstract}
Participants maneuvered a rat image through a circular region on the computer screen to find a hidden target platform, blending aspects of two well-known spatial tasks. Like the Morris water maze task, participants first experienced a series of learning trials before having to navigate to the hidden target platform from different locations and orientations. Like the dot-location task, they determined the location of a position within a two-dimensional circular region. This procedure provided a way to examine how the number of surrounding cues $(1,2$, or 3$)$ affects the memory for spatial location in navigation. Memory performance was better when there were more cues and when targets were close to cues, consistent with the idea that cues bolster fine-grain memory, especially in proximal regions. Early and late measures of bias in memory reflected biases in a direction toward the nearest cue, implicating a cue-based category structure of the navigational space. Collectively, results suggest cue-based spatial memory representations that have been inferred from the dot-location task generalize to a navigation task within a simple, computer-based environment, as demonstrated by the good fits of the spatial model developed for the dot-location task (Fitting, Wedell, \& Allen, 2005, 2007).
\end{abstract}

Keywords: Navigation; Spatial memory; Spatial reorientation; Mental rotation; Two-dimensional environment; Bias; Categorical coding; Spatial categories

\section{Introduction}

Spatial memory for object location has been studied extensively in various ways and across a variety of species (Morris \& Parslow, 2004; Nadel, 1990; Newcombe \& Huttenlocher, 2000). One simple experimental paradigm used to study spatial memory in

Correspondence should be sent to Sylvia Fitting, Department of Pharmacology \& Toxicology, Virginia Commonwealth University, Room 437, Kontos Medical Science Bldg., 1217 East Marshall Street, Richmond, VA 23298-0613. E-mail: sfitting@vcu.edu 
humans is the dot-location task, which requires participants to indicate remembered locations of dots within a circular task field (Fitting, Wedell, et al., 2007; Huttenlocher, Hedges, \& Duncan, 1991). Although memory for location in this task is generally very good, the observed biases in estimation have provided insights into the underlying spatial memory representation. The dot-location task has proven a successful aid in understanding spatial memory, but it is a very simple task and typically tests memory within seconds of learning the target location so that long-term spatial memory is not evaluated (for some exceptions to this, see Fitting, Allen, \& Wedell, 2007; Hund \& Plumert, 2002, 2005). Further, it evaluates memory for location at a single time point and thereby does not reflect the dynamically changing situation found in spatial navigation tasks.

In the research reported here, we incorporated a navigational component into the dotlocation task that enables us to consider how spatial memory representations may change as one progresses toward the target location. Because our task is mapped onto a small twodimensional computer display, the generality of the findings will be limited to navigation in small spaces over brief time periods. However, we believe this work provides an important conceptual link to the navigation literature and, in particular, to the well-known Morris water maze task (Morris \& Parslow, 2004), which is typically used to study spatial memory in animals. We will first briefly review the literature relevant to the dot-location task, then describe how we incorporated features of the Morris water maze task, and finally describe details of our adapted task, relevant measures, and the hypotheses that we will test.

\subsection{The dot-location task}

The dot-location task provided the impetus for the development of the category-adjustment model by Huttenlocher and colleagues (Huttenlocher, Hedges, Corrigan, \& Crawford, 2004; Huttenlocher et al., 1991; Huttenlocher, Hedges, Lourenco, Crawford, \& Corrigan, 2007). This model gives a compelling theoretical account of how biases arise in spatial representations. It is based on the idea that memory for spatial location is encoded at two levels: a fragile and briefly enduring fine-grain level that reflects analog veridical information, and a more robust categorical level that represents coarser and more discrete information. According to the category-adjustment model, bias in estimation results from the use of category prototypes to resolve uncertainty about fine-grain information, so that remembered locations shift toward the nearest prototype location. Although use of these categorical codes may bias estimates, they also serve the adaptive purpose of reducing the overall error in estimation. When the task field is circular, the fine-grain coordinate system is typically defined by polar coordinates and categories are typically defined by the four quadrants resulting from the imposition of vertical and horizontal axes onto the spatial environment. These quadrant-based categories are represented by category prototypes. Because exact memory of the location (fine-grain memory) fades rapidly, biases occur through the recruitment of category information that results in estimations being driven toward the central location of each category, or the category prototype.

Numerous studies have investigated how prototype locations are determined. Different factors have been hypothesized to influence the category structure, such as the distribution 
of spatial locations (Crawford, Margolies, Drake, \& Murphy, 2006; Hund \& Plumert, 2003; Spencer \& Hund, 2002), or the geometric configuration of the task field (Wedell, Fitting, \& Allen, 2007). A very influential determinant of the number and locations of prototypes that our current study builds on is the set of external cues located around the task field. In our recently reported experiments in this area (Fitting, Wedell, et al., 2007; Fitting, Wedell, \& Allen, 2008a, 2008b), participants reproduced the location of a dot in a circular region under conditions that varied the number and locations of cues placed along the perimeter of the task field. Results indicated that the category structure used to encode spatial location was highly dependent on the stability of one's orientation to the task field. When orientation was stable, the default four-quadrant category structure found in the Huttenlocher et al.'s (1991) study was used. This result held up even when the number of cues was increased dramatically (Fitting et al., 2008b). However, when orientation to the task field was shifted on a majority of trials, a cue-based category structure was adopted, in which available peripheral cues helped to define spatial prototypes used in locating the object. This cue dependence was reflected in the occurrence of very different patterns of bias for different cue conditions, with these patterns well described by our flexible fuzzy-boundary model (see Fitting, Wedell, et al., 2007; Fitting et al., 2008a). Our model was developed as an extension of Huttenlocher et al.'s (1991) category-adjustment model, with modifications that allow for inference of the number and locations of prototypes and how prototypes are recruited. In addition to bias effects on remembered location, we also observed strong cue effects on absolute memory error, consistent with the idea that cues serve to bolster the fine-grain information in regions proximal to the cue. Thus, the upshot of our recent research is that external cues may be very important in determining spatial categories in sparse and dynamically changing environments, such as when one navigates through a vista space, such as an arena, or even within a small spatial display on a computer screen.

We adapt our previous modeling methods for determining cue effects on spatial memory representations in the dot-location task to the navigation task we describe in the next section. Our modeling derives from four basic equations, which we present here using general terminology so that the reader can easily see the basis of the model. Specific adaptations of these equations are described in the Results section.

First, following Huttenlocher et al. (1991), we conceive of the participant's estimation of a given spatial location as a weighted average of two memory components, fine-grain memory and categorical memory, as represented by spatial prototypes. This basic relationship is described as follows:

$$
\text { Estimate }=\lambda(\text { Fine-grain memory location })+(1-\lambda)(\text { Relevant prototype }),
$$

where $\lambda$ represents the relative weight of the fine-grain information. Within this framework, the fine-grain memory location is presumed to be unbiased but fragile and $\lambda$ is assumed to decrease as memory for fine-grain information degrades.

The second equation derives from the first but focuses on bias, which is calculated by subtracting the actual location coordinates from the estimated location coordinates. The coordinate system used in modeling the data can be polar coordinates (Fitting, Wedell et al., 2007), in which case our primary concern is with angular bias and secondarily with radial 
bias, or it can be Cartesian coordinates (Wedell et al., 2007). Both of these representations are used in the current research. However, using general terminology, bias is predicted from Eq. 1 as follows:

$$
\begin{array}{r}
\text { Bias }=[\lambda(\text { Fine-grain memory location })+(1-\lambda)(\text { Relevant prototype })] \\
- \text { Actual location. }
\end{array}
$$

Inboth equations, we refer to the relevant prototype. A feature of the flexible fuzzyboundary model is that it allows for the probabilistic recruitment of different prototypes that may influence the bias. The basic idea is that the relevance of a given prototype is based on the proximity of the target location to the different prototypes. One consequence of this recruitment is that targets located near a border between two prototypes will tend to recruit both and hence have greatly diminished bias relative to that predicted by the standard category-adjustment model. Thus, relevance weighting is determined as follows:

$$
\text { Relevance of prototype }=\frac{\text { Proximity }(\text { Target }, \text { Prototype })}{\sum \text { Proximity }(\text { Target } \text { Prototypes })} \text {. }
$$

We have typically used an exponential decay function to describe proximity either using polar coordinates (Fitting, Wedell, et al., 2007; Fitting et al., 2008a, 2008b) or Cartesian coordinates (Wedell et al., 2007).

Finally, we have found it useful to model the error in fine-grain memory as a function of the distance from the target location to the cue locations (Fitting, Wedell, et al., 2007; Fitting et al., 2008a, 2008b). Although it is difficult to obtain a pure measure of fine-grain error, much of the variation in absolute error (i.e., the unsigned distance from estimate to actual location) is based on this source of error. The general form of the equation we use to model these error measures is as follows:

$$
\text { Fine-grain memory error }=\text { Baseline error }+b \text { (Distance to closest cues }),
$$

where $b$ indexes the additional error resulting from being located away from cues. The closeness of cues can be modeled using relevance weighting like that shown in Eq. 3. Note that Eq. 4 has two fundamental implications. First, it predicts that error will be decreased for targets located near cues. Second, it predicts that increasing the number of cues will reduce fine-grain error because targets will be more likely close to cues.

In summary, Eqs. 1-3 describe the flexible fuzzy-boundary model that we will apply to the bias data from our task to determine the nature of how cues influence the spatial categories represented in memory. Equations 3 and 4 will be applied to unsigned error measures that should relate primarily to fine-grain memory error and further explicate the role of cues. Thus, we believe our model-based analyses will promote a better theoretical understanding of the underlying mechanisms of spatial memory in our navigation task. 


\subsection{Integrating a navigational component into the task}

The standard navigation task used to assess spatial memory in animal research is the Morris water maze (Morris, 1981; Morris, Garrud, Rawlins, \& O’Keefe, 1982). Although water maze studies have typically used rats, there are now several studies that have investigated human participants in analogs of the water maze (Astur, Ortiz, \& Sutherland, 1998; Astur, Taylor, Mamelak, Philpott, \& Sutherland, 2002; Fitting, Allen, et al., 2007; Jacobs, Thomas, Laurance, \& Nadel, 1998; Parslow et al., 2005). Computer-generated environments are also increasingly popular as a tool to study place learning and memory (Jacobs, Laurance, \& Thomas, 1997; Maguire, Frith, Burgess, Donnett, \& O’Keefe, 1998; Moffat \& Resnick, 2002; Sandstrom, Kaufman, \& Huettel, 1998; Skelton, Bukach, Laurance, Thomas, \& Jacobs, 2000; Skelton, Ross, Nerad, \& Livingstone, 2006). The obvious advantages of this approach include that one can freely design environments according to study requirements, avoid the large costs in terms of effort, logistics, and control associated with studying behavior in vista space or large-scale environments, and precisely track participants' movements in the environment.

In addition to the examination of the neurobiological substrates of place learning and memory (Grön, Wunderlich, Spitzer, Tomczak, \& Riepe, 2000; Jarrard, 1993; Morris \& Parslow, 2004; O’Keefe \& Nadel, 1978; Sutherland \& Rudy, 1987), a number of environmental and experiential factors have been investigated to better understand the memory mechanisms underlying place learning and memory. Several studies have investigated the importance of cue availability in place learning (O'Keefe \& Nadel, 1978; Prusky, West, \& Douglas, 2000). Nadel et al. (1998) demonstrated that the removal of individual cues after learning did not impair performance at all unless all distal cues were removed. This finding is also confirmed in research with humans using a virtual world analog to the water maze task (Jacobs et al., 1998). These results seem very surprising given the reduction in accuracy one would expect when there are fewer than three cues available for the triangulation of location. However, it may be that, in these studies, available geometric features of the task field or other subtle distal cues were used to support the memory for location. Given this evidence, we believe that the basic question of whether more peripheral cues lead to increased accuracy during place learning in humans has yet to be well established.

We believe it is useful to combine aspects of the dot-location task and the Morris water maze navigation task to develop an intermediate task that provides further understanding of cue usage in navigation. We focused on three main adaptations of the dot-location task based on facets of the water maze task. First, the Morris water maze task includes a navigational component that allows one to trace the path to the target from start to finish. We modified the dot-location task to include this navigational component so that cue usage could be gauged by navigational measures related to (a) initial heading bias and error, (b) efficiency of the path taken, and (c) indicators of bias near the end of the search. Second, the water maze task consists of a learning phase in which the target platform's location is learned through exploration from a fixed orientation. Our adapted task includes this division between the learning and test phases. Finally, during the test phase, the orientation to the water maze is typically varied so that the subject cannot depend on egocentric cues but must 
use an allocentric encoding of the spatial location based on cues. Our adapted task included this manipulation of orientation to the task field.

\subsection{Adapted task}

Before clarifying our hypotheses, we believe it is instructive to describe essential elements of our navigation task. Fig. 1 presents the layout used for the task. Although the participant saw a white circular task field on a black background, the diagram in Fig. 1 presents the underlying grid structure through which participants navigated a rat image. As the participant moved the rat image continuously in search of one of the 16 target platform locations shown in Fig. 1, each successive location was recorded in row and column grid coordinates. Participants inferred the orientation of the task field relative to learning trials from the relative locations of the cues, with participants exposed to the same cues $(1,2$, or 3$)$ throughout the experiment.

Fig. 2 depicts the four rotations and four starting places used in the task, with an example target platform location. To test model-based predictions, we needed platform locations within each quadrant, at more than one radial distance, and with cue locations that would produce a pattern of bias different from the standard four-quadrant pattern. As depicted, the starting location of the rat image was at one of four positions (north, south, east, and west) and the task field was in one of four orientations $\left(0^{\circ}, 90^{\circ}, 180^{\circ}\right.$, or $\left.270^{\circ}\right)$. Fig. 2 shows the

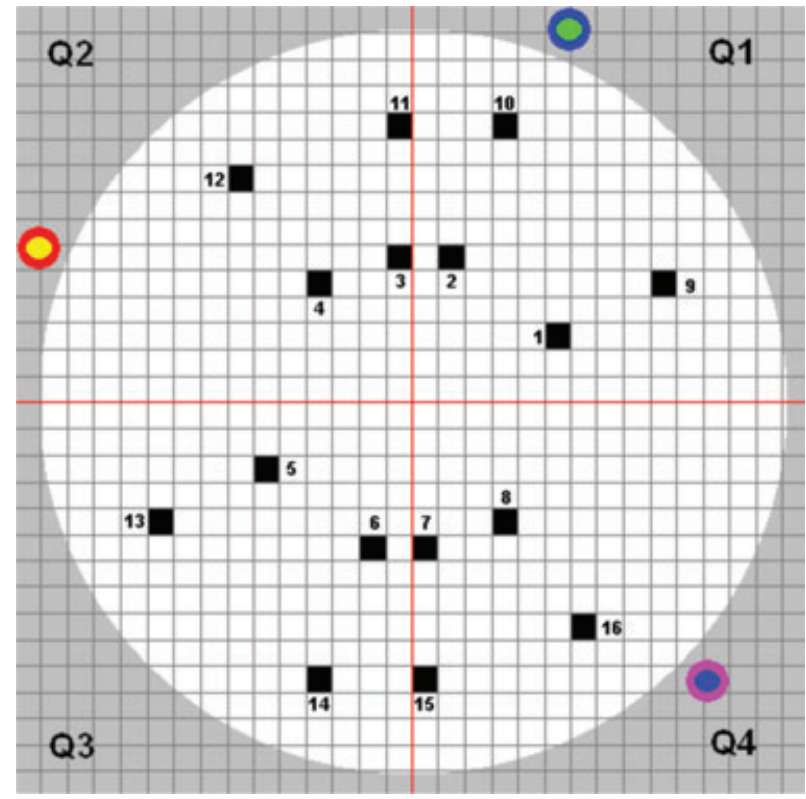

Fig. 1. Target platforms located at a short radius of 92 pixels from the center (target platforms from 1-8) and at a long radius of 168 pixels from the center (target platforms from 9-16). The overlaid grid was used to record participant's moves and was hidden during the actual test. The red horizontal and vertical lines divide the task field into four quadrants, that is, (Q)1, Q2, Q3, and Q4, and were not viewable. 


\section{Circle Orientation $-0^{\circ} \quad$ Circle Orientation $-90^{\circ}$}

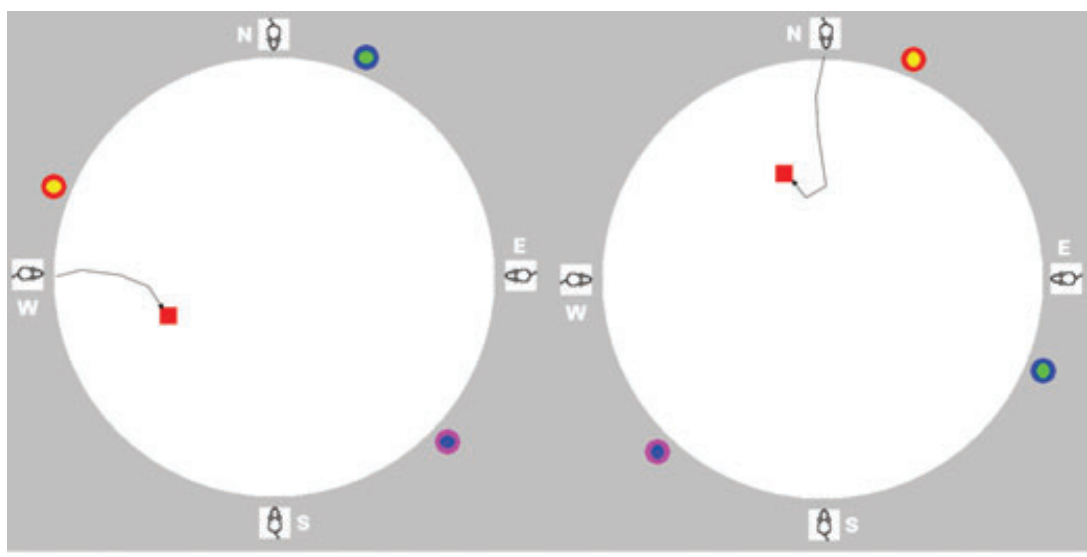

Circle Orientation $-180^{\circ} \quad$ Circle Orientation $-270^{\circ}$

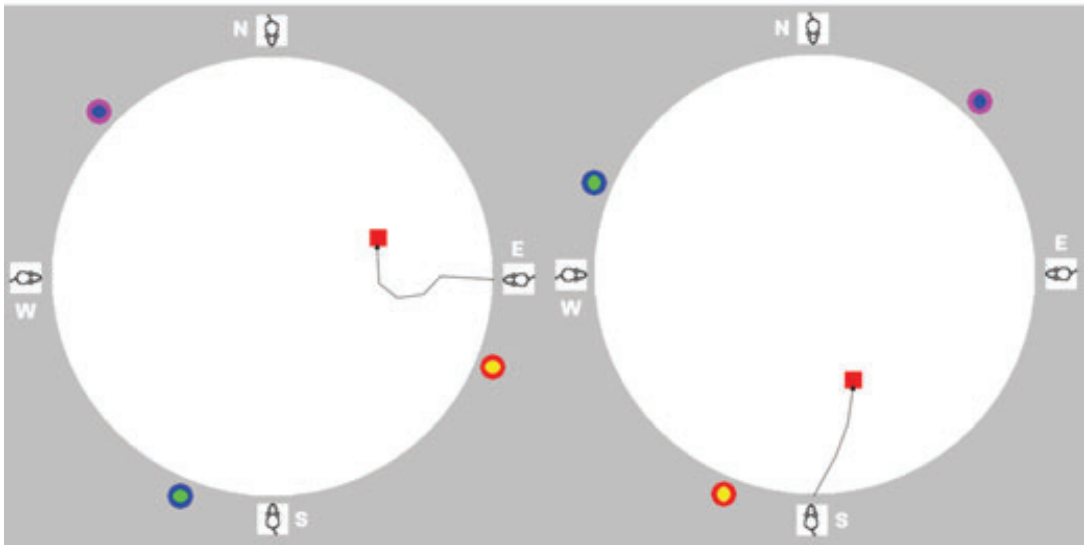

Fig. 2. Illustration of the circular task field from four different orientations $\left(0^{\circ}, 90^{\circ}, 180^{\circ}\right.$, and $\left.270^{\circ}\right)$ each indicated with four different entry points $(\mathrm{N}=$ north, $\mathrm{S}=$ south, $\mathrm{E}=$ east, and $\mathrm{W}=$ west $)$ and the three surrounding cue locations, a cue blue and violet in color located at $305^{\circ}$, a second cue green and blue in color located at $80^{\circ}$, and a third cue yellow and red in color located at $170^{\circ}$. The lines indicate examples of navigational pathways.

location of the same target platform relative to the cues for each of the four orientations. As in the water maze procedure, the orientation to the cues and the starting position were altered on test trials. Our main dependent variables were collected on these 16 test trials for each target platform, with the order of these trials randomized for each participant and for each target platform.

\subsection{Relevant measures for the adapted task}

Our focus in this study was on how memory for spatial location was affected by the cues presented outside the task field. In addition to production measures that are targeting specifically performance level of spatial tasks, we also looked at a variety of measures that more 
specifically targeted the process of spatial performance, which may be related to measures of discrimination tasks (see Velea, 2006). We used four dependent variables to analyze spatial memory processes and performance and describe each of these in turn.

\subsubsection{Excess moves}

This dependent variable was calculated by subtracting the minimum number of grid spaces required to navigate to the target platform from the actual number of traversed. Note that the maximum number of recorded moves was 200, as the trial was terminated if the target platform was not found after 200 grid space transitions. By subtracting the minimum required moves, we eliminate artifactual effects related to differences in path length between the starting location and the target location. This variable reflects the overall inefficiency in navigation and hence inaccuracy of place memory. It parallels the typical swimming time measures used in water maze tasks, in which greater swimming time reflects poorer memory. Our modeling of excess moves will be based on Eqs. 3 and 4.

\subsubsection{Initial heading error}

This dependent variable was calculated by recording the absolute angular difference between the true heading toward the target and the heading taken by a participant. The participant's heading was calculated based on the average of the column and row coordinates from the first 10 moves. This measure reflects the inaccuracy of the heading the participant first took and thereby early effects of cues on navigation. Again, our modeling of heading error will be based on Eqs. 3 and 4.

\subsubsection{Initial heading bias}

Heading was essentially calculated in the same way as initial heading error except that it was a signed variable, with the sign indicating whether the actual heading was counterclockwise to the target heading (positive values) or clockwise to the target heading (negative values) using polar coordinates. We signed heading bias in this way so that vectors headed toward the same location relative to the target would receive the same sign, regardless of the starting location. This measure allows us to compare the bias effects in initial heading with the bias effects predicted by our models developed for the dot-location task. Our modeling of initial heading bias is based on Eqs. 2 and 3 and utilizes polar coordinates to predict angular bias.

\subsubsection{End-position bias}

This dependent variable is based on the last 10 grid spaces traversed before reaching the target platform. Each move was recorded in row and column coordinates. End-position bias is calculated by subtracting the actual target location (in row and column coordinates) from the average of locations from the last 10 moves. This measure reflects the bias effects at the very end of the navigation process and thus late effects of cues on navigation. It should be noted that this measure only reflects bias effects when one collapses across starting position. Our modeling of end-position bias is based on Eqs. 1, 2, and 3 and utilizes Cartesian coordinates. 


\subsection{Three primary hypotheses}

We used the measures described above to assess three main sets of hypotheses, with specific hypotheses summarized in Table 1 . The first set of these hypotheses focused on effects of number of cues, the second on locations of targets relative to cues, and the third on effects of rotation. Our hypotheses were based on our assumption that participants would use the following simple navigation strategy. First, the participant would estimate a location for the hidden platform and attempt to move the rat icon directly to that location. Second, in the event that the location was not found, the participant would search in ever-widening radius of the estimated location.

\subsubsection{Number of cues}

Most of our work on cue-based bias has demonstrated that increasing the number of cues decreases the degree of categorical bias and the overall inaccuracy of memory (Fitting et al., 2005; Fitting, Wedell, et al., 2007; Fitting et al., 2008a). Theoretically,

Table 1

Delineation of three primary experimental hypotheses

\begin{tabular}{|c|c|}
\hline \multicolumn{2}{|c|}{ Hypotheses regarding number of cues } \\
\hline $\mathrm{H}_{1 \mathrm{a}}$ & $\begin{array}{l}\text { Increasing the number of cues will lead to a decrease in excess moves, reflected in a main effect of } \\
\text { cues }\end{array}$ \\
\hline $\mathrm{H}_{1 \mathrm{~b}}$ & $\begin{array}{l}\text { Increasing the number of cues will lead to a decrease in initial heading error, reflected in a main } \\
\text { effect of number of cues }\end{array}$ \\
\hline $\mathrm{H}_{1 \mathrm{c}}$ & $\begin{array}{l}\text { Increasing the number of cues will lead to a decrease in heading bias, reflected in an interaction of } \\
\text { number of cues and target angle }\end{array}$ \\
\hline $\mathrm{H}_{1 \mathrm{~d}}$ & $\begin{array}{l}\text { Increasing the number of cues will lead to a decrease in absolute end-position error, reflected in a } \\
\text { main effect of cues }\end{array}$ \\
\hline \multicolumn{2}{|r|}{ Hypotheses regarding proximity of cues } \\
\hline $\mathrm{H}_{2 \mathrm{a}}$ & $\begin{array}{l}\text { Memory for targets will be best when the target is located close to a cue, reflected in a main effect } \\
\text { of radius and a Cue } \times \text { Target Angle interaction for excess moves }\end{array}$ \\
\hline $\mathrm{H}_{2 \mathrm{~b}}$ & $\begin{array}{l}\text { Memory for targets will be best when the target is located close to a cue, reflected in a main effect } \\
\text { of radius and a Cue } \times \text { Target Angle interaction for initial heading error }\end{array}$ \\
\hline $\mathrm{H}_{2 \mathrm{c}}$ & $\begin{array}{l}\text { There will be an initial heading bias in the direction of the cue located closest to the target, } \\
\text { reflected in a main effect of target and a Cue } \times \text { Target interaction }\end{array}$ \\
\hline $\mathrm{H}_{2 \mathrm{~d}}$ & $\begin{array}{l}\text { There will be an end-position bias in the direction of the nearest cue-based prototype, reflected in a } \\
\text { Coordinate } \times \text { Target Angle and Coordinate } \times \text { Cue } \times \text { Target Angle interaction }\end{array}$ \\
\hline \multicolumn{2}{|r|}{ Hypotheses regarding rotation } \\
\hline $\mathrm{H}_{3 \mathrm{a}}$ & $\begin{array}{l}\text { Memory for targets will be best when the task field is at the same orientation as in learning, } \\
\text { reflected in a main effect of orientation on the excess moves measure }\end{array}$ \\
\hline $\mathrm{H}_{3 b}$ & $\begin{array}{l}\text { Memory for targets will be best when the task field is at the same orientation as in learning, } \\
\text { reflected in a main effect of orientation on the initial heading error measure }\end{array}$ \\
\hline $\mathrm{H}_{3 \mathrm{c}}$ & $\begin{array}{l}\text { Memory for targets will be best when the task field is at the same orientation as in learning, } \\
\text { reflected in an Orientation } \times \text { Target Angle interaction for initial heading bias }\end{array}$ \\
\hline $\mathrm{H}_{3 \mathrm{~d}}$ & $\begin{array}{l}\text { Memory for targets will be best when the task field is at the same orientation as in learning, } \\
\text { reflected in an orientation interaction for absolute end-position error }\end{array}$ \\
\hline
\end{tabular}


these effects derive from mechanisms related to the distance between the target location and the cue or prototype. In terms of bias, if cues represent prototypes, then bias increases as a function of the distance from the target to the cue, as described in Eq. 2. With more cues, targets will generally be less distant from the nearest cue-based prototype and hence the degree of bias will decrease. Second, even when cues are not used as prototypes, they appear to serve the function of anchoring fine-grain memory in regions proximal to the cue (e.g., Fitting et al., 2008b). Equation 4 captures this idea by the increase in fine-grain error as the distance to cues increases. These effects on fine-grain error also affect the degree of categorical bias, as the weighting of prototypes ( $\lambda$ in Eqs. 1 and 2 ) is assumed to increase with a decrease in fine-grain memory quality. With more cues, the distance to the nearest cue decreases on average and hence fine-grain memory should be bolstered and bias decreased. Our hypotheses assume that results from the dot-location task will apply throughout the navigation task. Thus, as described in Table $1, \mathrm{H}_{1 \mathrm{a}}$ predicts the reduced excess moves with more cues, $\mathrm{H}_{1 \mathrm{~b}}$ predicts the reduced heading error with more cues, $\mathrm{H}_{1 \mathrm{c}}$ predicts the reduced heading bias with more cues, and $\mathrm{H}_{1 \mathrm{~d}}$ predicts the reduced end-position error with more cues.

\subsubsection{Target proximity to cues}

Previous work has demonstrated that in the dot-location task, a fine-grain memory advantage exists for targets nearer the circumference and hence nearest to cues (Fitting, Wedell, et al., 2007; Fitting et al., 2008a). Those studies that involved rotation showed additional reduction in error as angular distance to the cues was reduced. We attribute both effects to the idea that cues serve to anchor fine-grain memory in regions proximal to the cues. This study examines whether this advantage exists in a navigation task and whether it can be found during early and late searching behavior. Again, our hypotheses assume that results from the dot-location task will apply throughout the navigation task. Thus, as described in Table $1, \mathrm{H}_{2 \mathrm{a}}$ predicts the reduced excess moves for targets proximal to cues, $\mathrm{H}_{2 \mathrm{~b}}$ predicts the reduced heading error for targets proximal to cues, $\mathrm{H}_{2 \mathrm{c}}$ predicts that the initial heading bias will be toward the nearest cue, and $\mathrm{H}_{2 \mathrm{~d}}$ predicts that the end-position bias will be toward the nearest cue-based prototype.

\subsubsection{Rotation}

In our previous work, we have observed that the participant's accuracy in the dotlocation task was best for $0^{\circ}$ rotation trials and became increasingly poorer as the angle of rotation increased (Fitting, Wedell, et al., 2007; Fitting et al., 2008a). This finding is consistent with the encoding specificity principle, in which memory is the best when context at retrieval matches context at encoding (Tulving \& Thomson, 1973). We assumed this finding from the dot-location task would apply again to the navigation task. Thus, as described in Table $1, \mathrm{H}_{3 \mathrm{a}}$ predicts the reduced excess moves for $0^{\circ}$ rotation, $\mathrm{H}_{3 \mathrm{~b}}$ predicts the reduced heading error for $0^{\circ}$ rotation, $\mathrm{H}_{3 \mathrm{c}}$ predicts the reduced initial heading bias for $0^{\circ}$ rotation, and $\mathrm{H}_{3 \mathrm{~d}}$ predicts the reduced endposition error for $0^{\circ}$ rotation. 


\section{Method}

\subsection{Participants}

Participants were 172 students from the University of South Carolina who volunteered in exchange for course credits. Data from 122 participants were retained for the analysis after dropping data from those who did not meet the learning criterion prior to test trials as described below.

\subsection{Task}

All participants attempted to navigate to the locations of eight hidden target platforms located within a circular region. The target platform is defined as the escape platform in the "real" Morris water maze task. The eight targets were distributed over the circular area in order to provide sufficient data for modeling the predicted biases and errors. Radius was manipulated as a between-subjects factor: For half the participants, the eight target platform trials, thus the eight hidden target platforms were located at a radius of 92 pixels (short radius; see Fig. 1, target platforms 1-8); and for the other half, the eight target platform trials, thus the eight hidden target platforms were located at a radius of 168 pixels (long radius; see Fig. 1, target platforms 9-16). Throughout the study, we will refer to these locations as the "target locations."

Of the eight target locations for short and long radius conditions, two were located within each of the four quadrants. Target platform trial blocks were presented successively in randomized blocks. Number of external reference cues was manipulated between subjects, with either one, two, or three cues along the circular region. Participants were randomly assigned to one of the six (Radius $\times$ Cue) conditions. Moves while navigating through the computerbased environment were recorded in $x$ and $y$ coordinates using the overlaid grid illustrated in Fig. 1.

Table 2 provides detailed descriptions of the 16 target locations, identified by their radius value (short or long) and the quadrant in which they reside. Angle is based on polar coordinates (from the center of the circle) and Q-angle reflects the angular location within the quadrant. Headings 1-4 indicate the angular heading from the four different starting locations, used in computing the heading error and bias. Finally, the minimum moves from each starting position to each target platform were used to determine excess moves.

\subsection{Materials and apparatus}

All materials and instructions were presented on desktop computers with 15 -in $(38-\mathrm{cm})$ monitors. Participants' distance to the monitor was between 30 and $60 \mathrm{~cm}$. The white circular region was presented on a black background with a radius of 220 pixels in video graphics array mode at a resolution of $640 \times 480$ pixels. An invisible grid with 30 rows as the $y$ coordinate ( 17 pixels in width) and 30 columns as the $x$ coordinate (17 pixels in height) divided the circular area and was used to measure moves while navigating through the circular field. 
Table 2

Location, initial heading, and minimum move information for targets

\begin{tabular}{lrrrrrrrrrrrrrrrr}
\hline Target & 1 & 2 & \multicolumn{1}{c}{3} & \multicolumn{1}{c}{4} & \multicolumn{1}{c}{5} & 6 & 7 & 8 & 9 & 10 & 11 & 12 & 13 & 14 & 15 & 16 \\
\hline Radius & $\mathrm{S}$ & $\mathrm{S}$ & $\mathrm{S}$ & $\mathrm{S}$ & $\mathrm{S}$ & $\mathrm{S}$ & $\mathrm{S}$ & $\mathrm{S}$ & $\mathrm{L}$ & $\mathrm{L}$ & $\mathrm{L}$ & $\mathrm{L}$ & $\mathrm{L}$ & $\mathrm{L}$ & $\mathrm{L}$ & $\mathrm{L}$ \\
Quadrant & 1 & 1 & 2 & 2 & 3 & 3 & 4 & 4 & 1 & 1 & 2 & 2 & 3 & 3 & 4 & 4 \\
Angle & 25 & 73 & 95 & 130 & 205 & 253 & 275 & 310 & 25 & 73 & 95 & 130 & 205 & 253 & 275 & 310 \\
Q-angle & 25 & 73 & 5 & 40 & 25 & 73 & 5 & 40 & 25 & 73 & 5 & 40 & 25 & 73 & 5 & 40 \\
Heading 1 & 293 & 276 & 264 & 248 & 251 & 264 & 270 & 279 & 312 & 307 & 256 & 221 & 242 & 261 & 270 & 285 \\
Heading 2 & 6 & 17 & 20 & 20 & 342 & 335 & 338 & 345 & 10 & 29 & 36 & 45 & 315 & 315 & 324 & 337 \\
Heading 3 & 74 & 87 & 93 & 102 & 117 & 103 & 90 & 73 & 65 & 83 & 92 & 107 & 135 & 135 & 90 & 45 \\
Heading 4 & 168 & 159 & 162 & 168 & 189 & 201 & 203 & 204 & 141 & 138 & 146 & 159 & 192 & 211 & 218 & 228 \\
Min. Move 1 & 12 & 9 & 9 & 10 & 17 & 20 & 20 & 19 & 10 & 4 & 4 & 7 & 19 & 25 & 25 & 23 \\
Min. Move 2 & 20 & 16 & 14 & 11 & 9 & 13 & 15 & 18 & 24 & 18 & 14 & 8 & 5 & 11 & 15 & 21 \\
Min. Move 3 & 17 & 20 & 20 & 19 & 12 & 9 & 9 & 10 & 19 & 25 & 25 & 23 & 10 & 4 & 4 & 7 \\
Min. Move 4 & 9 & 13 & 15 & 18 & 20 & 16 & 14 & 11 & 5 & 11 & 15 & 21 & 24 & 18 & 14 & 8 \\
\hline
\end{tabular}

Note: S, Short; L, long; Q-angle, angle within quadrant; all heading and distances calculated using the up orientation, with numbers indicating starting position $(1=$ north, $2=$ west, $3=$ south, $4=$ east $)$. Heading: deviation from the starting point to the target location in degree; rounding value min. moves: minimum number of moves from the starting position to the target.

This grid is shown in Fig. 1, although it was not visible to participants. For each block of trials, a hidden target platform was located at a fixed position within the circle (i.e., for the short and the long radius the eight target locations were located at $25^{\circ}, 73^{\circ}, 95^{\circ}, 130^{\circ}, 205^{\circ}$, $253^{\circ}, 275^{\circ}$, and $310^{\circ}$ ). Across trial blocks, participants were asked to find each of the eight hidden target platform locations from four different entry points (north, south, east, and west) and from four different circle orientations $\left(0^{\circ}, 90^{\circ}, 180^{\circ}\right.$, and $\left.270^{\circ}\right)$. Starting locations and orientation manipulations are shown in Fig. 2.

In the one-cue condition, the reference cue (blue and violet in color) was located at $305^{\circ}$ along the outside of the circle; in the two-cue condition, an additional cue (green and blue in color) was located at $80^{\circ}$; and in the three-cue condition, an additional cue (yellow and red in color) was located at $170^{\circ}$ (Figs. 1 and 2). The colors for the cues were chosen to make them highly distinguishable from each other.

\subsection{Procedure}

Groups of 1-5 participants were individually tested on the computer. After reading the general instructions, participants were presented practice trials to move the rat image in the circular field. The rat image appeared just outside of one of the four entry locations, as shown in Fig. 2. To navigate with the rat to find the hidden target platform, the participant placed the cursor on the rat image, then held down the left mouse button and moved the rat image within the circular region. The rat image was able to be moved in any of eight directions (to the next grid location) within the circular field. Each successive move within the underlying grid of Fig. 1 was recorded. ${ }^{1}$ Following the initial practice session, participants were asked to navigate through the circular task field and find a hidden target platform that 
would be shown in red once found or once exceeding the maximum number of moves. The target platform was shown on the screen for $5 \mathrm{~s}$ and then covered by a dynamic checkerboard mask for $1.5 \mathrm{~s}$ followed by a blank circle, indicating the start of the next trial. The checkerboard mask consisted of white and black $10 \times 10$ pixels quadrants covering the circular region. The colors of the checkerboards were exchanged six times after $0.25 \mathrm{~s}$, creating a moving pattern to avoid fixation.

These procedures were illustrated to the participant with three preliminary sets of trials that included elaborate visual feedback demonstrations to make sure the task was understood. These were then followed by the eight blocks of experimental trial sets (one for each target platform). At the beginning of each set, there were four learning trials in which the participant attempted to find the target platform from the same $0^{\circ}$ orientation but from four different starting locations. Orientation was not varied on learning trials so that the participants would have an easier time learning the target location. The four learning trials were followed by 16 test trials in which each of the Orientation $\times$ Starting location conditions was presented in a random order. Thus, 160 trials (4 learning trials plus 16 testing trials for each of 8 target locations) were included in the experimental phase. Successive moves were recorded throughout navigation.

\subsection{Learning criteria}

Because our focus is only on those who had adequately learned the target location in the $0^{\circ}$ orientation, we established a learning criterion used to drop participants from the data analysis. The mean number of moves across the eight target locations for each of the four learning trials was calculated to determine the typical learning curve across participants. Based on this analysis, participants had to average $<46$ moves to find the target in the third learning trial and $<41$ moves in the fourth trial to meet the criteria. Note that for a given target platform and starting location, the minimum number of moves to the target ranged from 4 to 25 moves. We deemed that averaging more than 45 moves on the third trial would indicate a failure of learning. We lowered the criterion to 40 moves for the fourth trial to reflect the additional learning that was expected.

All data analyses we report are on the 122 participants who met the criteria. The number of participants retained in the six between-subjects conditions was $n=20$ for all but two conditions: $n=21$ in both the two-cue long-radius condition and three-cue short radius condition.

\section{Results}

For each of the dependent variables, factorial analyses of variance (ANOVAs) were conducted based on two between-subjects factors and three or four within-subjects factors. The between-subjects factors were cue condition (one, two, or three cue) and radius (short or long). Within-subjects factors were target angle (eight levels), grid orientation $\left(0^{\circ}, 90^{\circ}\right.$, $180^{\circ}$, and $270^{\circ}$ ), and starting position (north, south, east, and west). Because analysis of 
end-position bias was conducted on Cartesian coordinates, it included the additional withinsubjects factor of dimension ( $x$ and $y$ ). For the within-subjects factor, violations of compound symmetry were addressed via the use of the Greenhouse-Geisser degrees of freedom correction factor where applicable (Greenhouse \& Geisser, 1959).

\subsection{Excess moves}

Fig. 3 plots excess moves as a function of radius, target angle, and cue. The data are represented by symbols and model fits (described below) by functions, with arrows indicating cue locations. As shown in Fig. 3, there were systematic effects on excess moves related to radius, angle and cue. A 3 (Cue) $\times 2$ (Radius) $\times 8$ (Target Angle) $\times 4$ (Orientation) $\times 4$ (Start) mixed factorial ANOva was used to analyze excess moves. The corresponding column of Table 3 summarizes tests from that analysis, excluding tests of main effects of and interactions with orientation and starting position.

The significant main effect of cue was essentially completely described by its linear trend, $F(1,116)=28.58, p<.001$. Excess moves decreased systematically with an increase in the number of cues $M_{1}$ cue $=39.53, M_{2}$ cues $=28.63$, and $M_{3 \text { cues }}=22.50$ and supported $\mathrm{H}_{1 \mathrm{a}}$. Pairwise comparisons using Tukey's correction indicated that the one-cue condition significantly differed from the two-cue condition $(p<.01)$ as well as from the three-cue condition $(p<.001)$. Note also that the standard deviation decreased as well, with this decrease significant at $p<.05$ by an $F$-max test: $S D_{1}$ cue $=20.10, S D_{2}$ cues $=14.17$, and $S D_{3}$ cues $=8.52$. The violation of heterogeneity makes the ANOva test for this condition suspect. However, this effect was very robust so that even if we use the largest within-cell variance as a substitute for the error term, the effect is significant at $p<.01$.

The significant radius effect reflected greater difficulty in finding short radius targets $(M=34.53, S D=17.74)$ than long radius targets $(M=25.76, S D=13.82)$. This finding supported $\mathrm{H}_{2 \mathrm{a}}$, which asserted that short radius targets will be harder to find than long radius
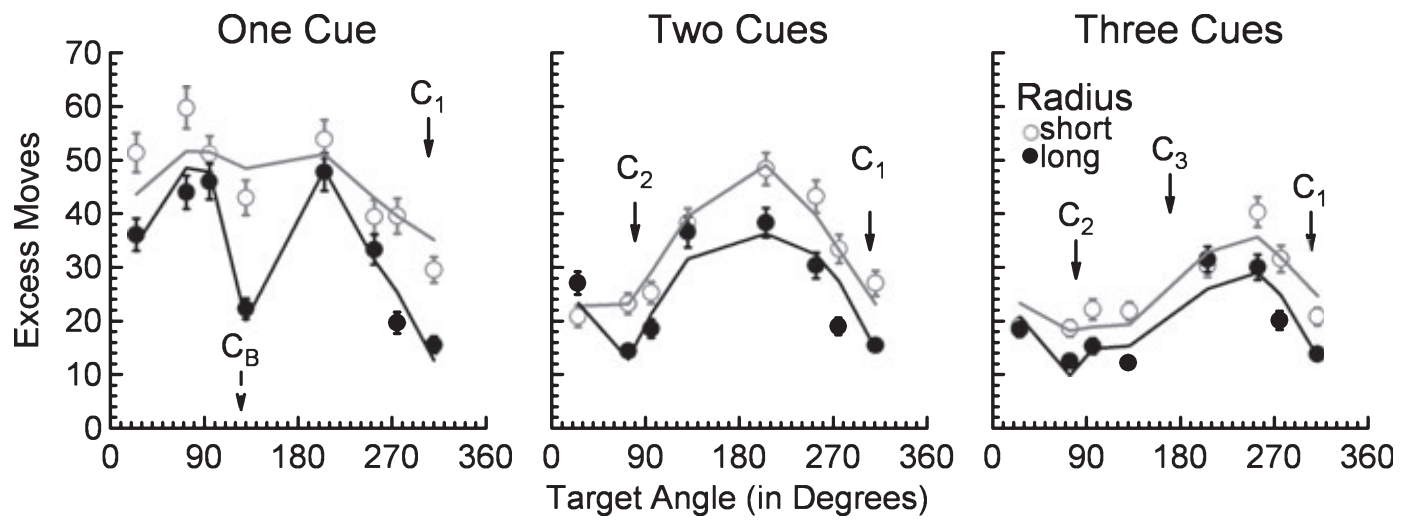

Fig. 3. Model fit based on Eq. 4a (using metric distance) on excess moves across target angle separate for the three cue conditions. The error bars indicate 1 standard error of the mean. $C_{\mathrm{B}}=$ boundary-based cue, $C_{1}=$ cue $1, C_{2}=$ cue 2 , and $C_{3}=$ cue 3 . 
Table 3

Degrees of freedom and $F$-values for 3 (Cue) $\times 2$ (Radius) $\times 8$ (Target Angle) $\times 4$ (Orientation) $\times 4$ (Start Position) mixed factorial ANOvAs

\begin{tabular}{lcccc}
\hline Source & $\mathrm{df}$ & Excess Moves & Initial Heading Error & Initial Heading Bias \\
\hline Cue $(C)$ & 2,116 & $14.59^{* * *}$ & $10.24^{* * *}$ & 2.21 \\
Radius $(R)$ & 1,116 & $11.66^{* * *}$ & $16.07^{* * *}$ & 1.00 \\
$R \times C$ & 2,116 & 0.59 & 0.21 & 0.61 \\
Target Angle $(\mathrm{TA})$ & 7,812 & $21.70^{* * *}$ & $14.38^{* * *}$ & $2.39^{*}$ \\
TA $\times C$ & 14,812 & $12.27^{* * *}$ & $6.18^{* * *}$ & $3.92^{* * *}$ \\
TA $\times R$ & 7,812 & 2.04 & $3.62^{* *}$ & $3.24^{* *}$ \\
TA $\times C \times R$ & 14,812 & 1.39 & $2.09^{*}$ & 1.43 \\
\hline
\end{tabular}

Note: $* p<.05, * * p<.01, * * * p<.001$, for the within-subjects factors: $p_{\mathrm{GG}}$, tests involving Orientation and Starting Position are not shown.

targets and confirmed previous research with reflecting a fine-grain memory advantage for targets nearer the circumference (Fitting, Wedell, et al., 2007; Werner \& Diedrichsen, 2002). This effect is shown in Fig. 3 by the systematically lower values of the filled circles (long radius targets) than the open circles (short radius targets).

The significant main effect of target angle indicated that some targets led to greater excess moves than others. In particular, excess moves were highest for target angles in quadrant 3, in which no cue was located. The significant Target Angle $\times$ Cue interaction reflects the negative relationship between target proximity to the cues and excess moves as shown in Fig. 3. When there is just one cue at $305^{\circ}$, excess moves are generally high except in quadrant 4 where the cue is located (and to some extent for the target location directly opposite the cue location). Likewise, excess moves are most reduced in quadrants 1 and 4 in the two-cue condition, as these are the quadrants where cues were located. Finally, the threecue condition demonstrates this same relationship, with excess moves greatest in quadrant 3 , which had no corresponding cue. This pattern of results supports $\mathrm{H}_{2 \mathrm{a}}$, which asserted that memory for target locations would be the best when the target is located close to a cue.

To better understand these relationships and test the idea that cues anchor fine-grain memory, the data were fit to a version of Eq. 4, substituting excess moves for fine-grain memory error and including a simplified version of Eq. 3 to determine relevance weighting. ${ }^{2}$ The general nonlinear regression equation we used to fit data within each cue condition was as follows:

$$
\mathrm{EM}_{i j}=b_{0 j}+\sum \frac{r_{i j k}}{b_{j k}} D_{i j k},
$$

where $\mathrm{EM}_{i j}$ is the excess moves for target angle $i$, radius $j, b_{0 j}$ is a baseline error value that can vary with radius, $b_{j k}$ weights distance from the target to cue $k$ and can vary with cue and radius, $D_{i j k}$ is the Euclidean distance between the target and the cue, and $r_{i j k}$ moderates weights and reflects the relevance or proximity of the target to the cue. The relevance weight was based on a negative exponential similarity function with a single parameter as follows: 


$$
r_{i j k}=\exp \left(-c D_{i j k}\right),
$$

where $c$ is a sensitivity or scaling parameter. Equation 3 a was substituted for $r_{i j k}$ in Eq. $4 \mathrm{a}$ when fitting the data using a least-squared error criterion. Based on our earlier modeling of the dot-location task (Fitting et al., 2008a), we included a boundary-based cue, with weight $b_{\mathrm{B}}$, in the one-cue condition located $180^{\circ}$ from the actual cue and that we have previously labeled a "phantom cue." Conceptually, the phantom cue might arise by participants projecting a cue location opposite the actual cue and using that as a guide to locate targets that are far from the single cue.

The model minimally requires three fitted parameters, $b_{0}, b$, and $c$. Higher parameter versions of the model allow the intercept to vary with radius and the weights to vary with cue or radius. We began modeling the data in each cue condition by fitting the highest parameter model and then successively constraining it by holding parameters constant that did not cause a significant drop in $R^{2}$. Table 4 presents the parameter values and $R^{2}$ values from these regressions. Although the use of distance to closest cues means that short radius targets will generally have higher error than long radius targets, only the fit to the one-cue condition sufficiently accounted for this effect without fitting additional parameters to radius. The one-cue condition was also the only condition that required that weights vary with cues. However, this is consistent with our previous work in which the phantom cue is not necessarily treated the same as actual cues (Fitting et al., 2008a). Overall, the fits of the four- to five-parameter models indexed $R^{2}$ values that were larger than .80 . Additionally, $R^{2}$ was significantly decreased in the one-cue condition if the phantom cue distance was not included, providing further support for a phantom cue at the boundary in the one-cue condition.

The fit of the model can be also visually assessed by examining how well the functions in Fig. 3 predict the variations in the data points. The prediction functions clearly capture the salient features of the data. The model captures the great reduction in excess moves when a target is located close to a cue. The greater sensitivity to cue locations for the long radius targets in the one-cue condition when compared with two- and three-cue conditions

Table 4

Parameter values and fit indices modeling excess moves for each of the three cue conditions

\begin{tabular}{|c|c|c|c|c|c|c|c|c|}
\hline & \multirow[b]{2}{*}{ Model } & \multirow[b]{2}{*}{ Radius } & \multicolumn{6}{|c|}{ Parameters } \\
\hline & & & $c$ & $b_{0}$ & $b_{1}$ & $b_{2}$ or $b_{\mathrm{b}}$ & $b_{3}$ & $R^{2}$ \\
\hline \multirow[t]{2}{*}{ Excess Move 1} & \multirow[t]{2}{*}{$4 \mathrm{P}$} & Short & 0.078 & -87.7 & 0.208 & 0.040 & - & .884 \\
\hline & & Long & 0.078 & -87.7 & 0.208 & 0.040 & - & \\
\hline \multirow[t]{2}{*}{ Excess Move 2} & \multirow[t]{2}{*}{$5 \mathrm{P}$} & Short & 0.042 & -154.3 & 0.084 & 0.084 & - & .888 \\
\hline & & Long & 0.042 & -43.6 & 0.218 & 0.218 & - & \\
\hline \multirow[t]{2}{*}{ Excess Move 3} & \multirow[t]{2}{*}{$5 \mathrm{P}$} & Short & 0.040 & -215.5 & 0.101 & 0.101 & 0.101 & .843 \\
\hline & & Long & 0.040 & -78.0 & 0.243 & 0.243 & 0.243 & \\
\hline
\end{tabular}

Note: $\mathrm{P}=$ parameter; $c=$ sensitivity parameter; $b_{0}=$ intercept; $b=$ weight of the distance from target to cue (subscripts indicate cue); bold faced values are free to vary; italicized values were constrained to be equal to a bold faced value. 
is modeled by a higher sensitivity parameter in the one-cue condition. The greatly reduced error at the location opposite the cue in the one-cue condition provides strong evidence for a boundary-based phantom cue. Overall, the modeling reinforces the importance of proximity to cues as a major factor guiding the efficiency of search.

In addition to the effects illustrated in Table 3 , a significant main effect of orientation was noted, $F(3,348)=62.26, p<.001$, reflecting reduced excess moves for $0^{\circ}$ orientation trials $(M=22.00, S D=13.65)$ compared with rotated trials $\left(M_{90^{\circ}}=33.00, S D=19.38\right.$; $\left.M_{180^{\circ}}=31.73, S D=19.10 ; \quad M_{270^{\circ}}=33.85, S D=19.00\right)$. Pairwise comparisons using Tukey's correction demonstrated that the only significant differences in excess moves were between the $0^{\circ}$ orientation and each of the other orientations. The finding that excess moves were significantly reduced in the $0^{\circ}$ orientation trials compared with the rotated orientation trials supports $\mathrm{H}_{3 \mathrm{a}}$, reflecting a memory advantage when testing in the orientation that matched the orientation during learning, an encoding specificity effect.

Additionally, the Orientation $\times$ Cue interaction was significant, $F(6,348)=9.59$, $p<.001$. Fig. 4 presents a bar graph of the relevant means making up the interaction. As is readily apparent from Fig. 4, the effect of cue is much larger for the three rotated orientations than for the nonrotated $0^{\circ}$ orientation. This effect is captured in the interaction contrast comparing the linear effect of cues in the learning orientation with that in the other three conditions, $F(1,119)=48.25, p<.001$. Importantly, while the linear effect of cue is reduced for the learning orientation, it is still significant, $F(1,119)=4.41, p<.05$, indicating that even in a task field orientation in which cues do not have to be used necessarily, an increase in the number of cues reflects better memory. This finding parallels our results using the dot-location task (Fitting, Wedell, et al., 2007). ${ }^{3}$

\subsection{Initial heading error}

Fig. 5 plots initial heading error as a function of radius, target angle, and cue. The data are represented by symbols and model fits (described below) by functions, with arrows

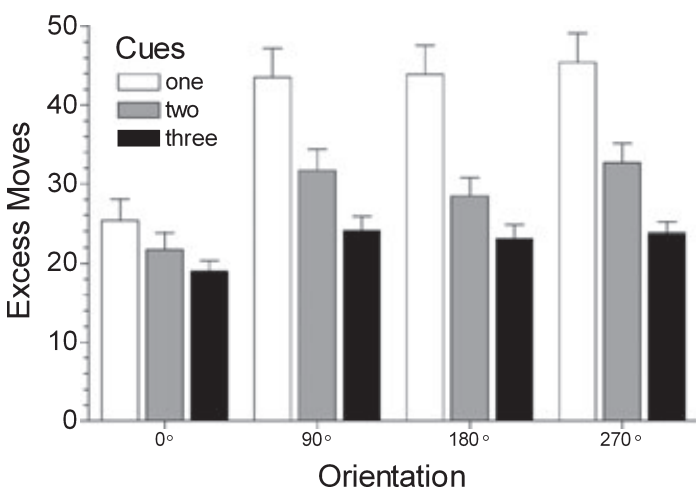

Fig. 4. Excess moves for the three cue conditions separate for orientation. The error bars indicate 1 standard error of the mean. A significant Orientation $\times$ Cue interaction was noted. 

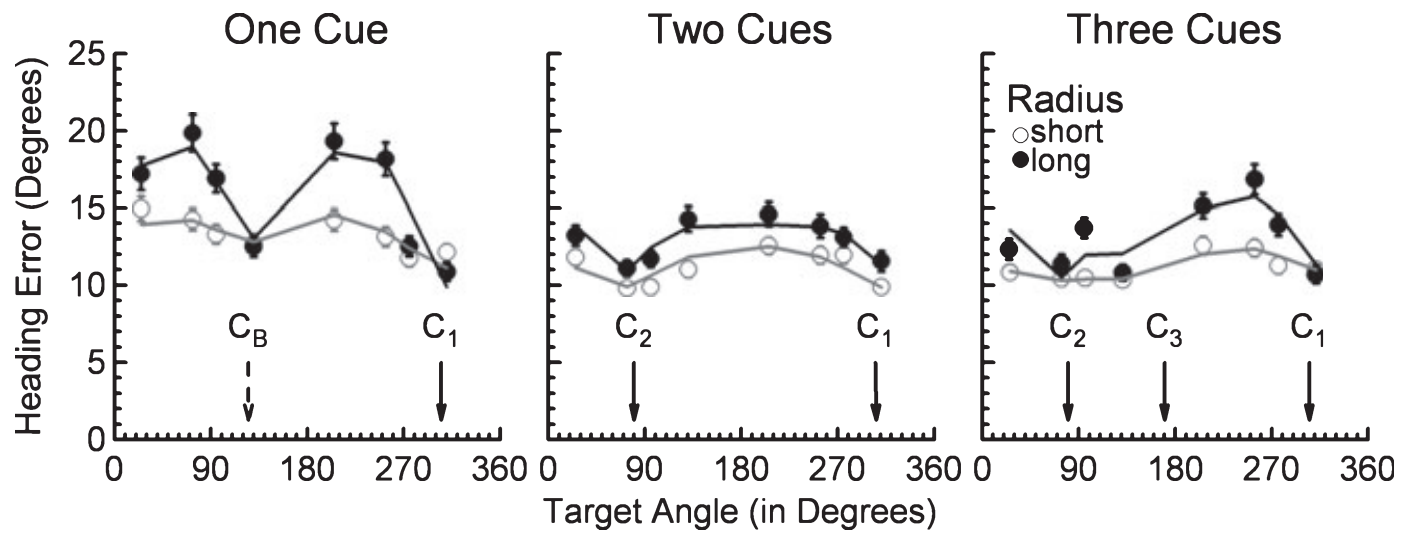

Fig. 5. Model fit based on Eq. 4b (using metric distance) on initial heading error across target angle separate for the three cue conditions. The error bars indicate 1 standard error of the mean. $C_{\mathrm{B}}=$ boundary-based cue, $C_{1}=$ cue $1, C_{2}=$ cue 2 , and $C_{3}=$ cue 3 .

indicating cue locations. As shown in Fig. 5, there were systematic effects on heading error related to radius, angle, and cue. A parallel $3 \times 2 \times 8 \times 4 \times 4$ mixed factorial ANOvA was used to analyze the initial heading error. The corresponding column of Table 3 summarizes tests from that analysis, excluding tests of main effects of and interactions with orientation and starting position.

The significant main effect of cues supported $\mathrm{H}_{2 \mathrm{~b}}$, indicating less initial heading error as the number of cues increased. Pairwise comparisons using Tukey's correction indicated that the one-cue condition $(M=14.64, S D=3.77)$ significantly differed from the two-cue condition $(M=12.07, S D=2.77)$ as well as from the three-cue condition $(M=12.14$, $S D=2.53)$. As with the excess moves measure, initial heading error did not decrease significantly from two to three cues.

There was a significant main effect of radius, with reduced initial heading error for the short radius targets $(M=11.88, S D=2.26)$ than for long radius targets $(M=13.99$, $S D=3.76$ ). This result is in the opposite direction of the effect reported for excess moves and considered in hypothesis $\mathrm{H}_{2 b}$, as short radius targets are typically more distant from the nearest cue than long radius targets. One explanation for this difference is that there was a tendency for participants to initially head toward the center of the circle, reflecting a center bias that would result in less heading error for short than for long radius targets. To investigate this explanation, we conducted additional analyses on center heading bias, calculated by coding the heading error toward the center as positive and the heading error away from the center as negative. A one-sample $t$ test on this measure indicated a significant positive bias, $M=5.01, S D=3.59, t(121)=15.42, p<.001$, confirming a bias toward the center of the task field. The tendency to head toward the center might then explain the significant radius effect for initial heading error due to the fact of creating more bias for long than for the short radius. This explanation is supported by results of a 3 (Cue) $\times 2$ (Radius) model ANOVA on center heading bias. A significant radius effect, $F(1,116)=39.12, p<.001$, 
reflected greater center heading bias for the long radius target $(M=6.74, S D=3.82)$ than for the short radius target $(M=3.28, S D=2.31)$. There was also a significant effect of cues, $F(2,116)=5.42, p<.01$, consistent with a greater center heading bias in the one-cue than the two- or three-cue condition.

Returning to the analysis of initial heading error, Table 3 also shows a large and significant main effect of target angle. Consistent with hypothesis $\mathrm{H}_{2 \mathrm{~b}}$, heading error was the greatest for targets in quadrant 3, which had no cues associated with it (Fig. 5). Target angle also interacted with cue and radius in significant two- and three-way interactions. These effects appear to be mostly captured by the idea that initial heading error is minimized for targets located near cues, as shown in Fig. 5.

To test this assertion and better understand these relationships, the data were fit to a version of Eq. 4 that paralleled the equation used for excess moves as follows:

$$
\mathrm{HE}_{i j}=b_{0 j}+\sum \frac{r_{i j k}}{b_{j k}} D_{i j k}
$$

with the only difference between Eqs. $4 \mathrm{a}$ and $4 \mathrm{~b}$ being the dependent measures $\left(\mathrm{HE}_{i j}\right.$ reflects the heading error for target angle $i$ and radius $j$ ). Parallel to our modeling of excess moves, we included a phantom cue for the one-cue condition in modeling the heading error. Equation $3 \mathrm{a}$ was substituted in for $r_{i j k}$ in Eq. $4 \mathrm{~b}$ when fitting the data using a least-squared error criterion. As with our earlier modeling, we began by fitting the highest parameter model and then successively constraining it by holding parameters constant that did not cause a significant drop in $R^{2}$. Table 5 presents the parameter values and $R^{2}$ values for the best-fit models. The selected models paralleled those for excess moves. In this case, however, the differences in the parameters tied to radius were needed to account for the greater heading error for long radius targets than for short radius targets. Once again, the fits of the four- to five-parameter models indicated $R^{2}$ values that were larger than .80 .

The fit of the model can be visually assessed in Fig. 5. Similar to the fit for excess moves, the model captures the great reduction in initial heading error when a target is located close

Table 5

Parameter values and fit indices modeling initial heading error for each of the three cue conditions

\begin{tabular}{|c|c|c|c|c|c|c|c|c|}
\hline & \multirow[b]{2}{*}{ Model } & \multirow[b]{2}{*}{ Radius } & \multicolumn{6}{|c|}{ Parameters } \\
\hline & & & $c$ & $b_{0}$ & $b_{1}$ & $b_{2}$ or $b_{\mathrm{b}}$ & $b_{3}$ & $R^{2}$ \\
\hline Initial heading & $4 \mathrm{P}$ & Short & 0.005 & -22.1 & 0.782 & 0.902 & - & .905 \\
\hline Error 1 & & Long & 0.005 & -22.1 & 0.782 & 0.902 & - & \\
\hline Initial heading & $5 \mathrm{P}$ & Short & 0.059 & -35.6 & 0.255 & 0.255 & - & .878 \\
\hline Error 2 & & Long & 0.059 & -0.2 & 0.832 & 0.832 & - & \\
\hline Initial heading & $5 \mathrm{P}$ & Short & 0.042 & -18.9 & 0.787 & 0.787 & 0.787 & .819 \\
\hline Error 3 & & Long & 0.042 & -13.9 & 0.846 & 0.846 & 0.846 & \\
\hline
\end{tabular}

Note: $\mathrm{P}=$ parameters; $c=$ sensitivity parameter; $b_{0}=$ intercept; $b=$ weight of the distance from target to cue (subscripts indicate cue); bold faced values are free to vary; italicized values were constrained to be equal to a bold faced value. 
to a cue and shows a clear effect of the phantom cue. In contrast to excess moves, there is a greater heading error for long radius targets than for short radius targets, which may be plausibly attributed to a bias to head toward the center of the task field. Overall, the modeling reinforces the importance of proximity to cues as a major factor guiding the efficiency of search.

In addition to the effects illustrated in Table 3, significant effects for orientation were noted: Out of 16 effects, 7 were significant at an $\alpha$-level of .01. Further, significant effects for starting location were noted: Out of 16 effects, 6 were significant at an $\alpha$-level of .01. ${ }^{4}$ Not surprisingly, these results indicate that both orientation to the circular task field and starting location influence the initial heading error. Because the focus of this research is primarily on effects of cues, we will not consider these further except to note that the main effect of orientation was consistent with hypothesis $\mathrm{H}_{3 \mathrm{~b}}$ in that the heading error was least for the $0^{\circ}$ orientation $(M=12.17, S D=3.77)$ compared with rotated trials $\left(M_{90^{\circ}}=13.84\right.$, $\left.S D=3.76 ; M_{180^{\circ}}=12.75, S D=3.29 ; M_{270^{\circ}}=12.98, S D=4.01\right)$, indicating an encoding specificity effect.

\subsection{Initial heading bias}

Fig. 6 plots the initial heading bias as a function of radius, target angle, and cue. The data are represented by symbols and model fits (described below) by functions, with arrows indicating cue locations. As shown in Fig. 6, there were systematic effects on heading bias related to radius, angle, and cue. A parallel $3 \times 2 \times 8 \times 4 \times 4$ mixed factorial ANOvA was used to analyze the initial heading bias. The corresponding column of Table 3 summarizes tests from that analysis, excluding tests of main effects of and interactions with orientation and starting position. The main effect of target angle indicates that the initial heading bias
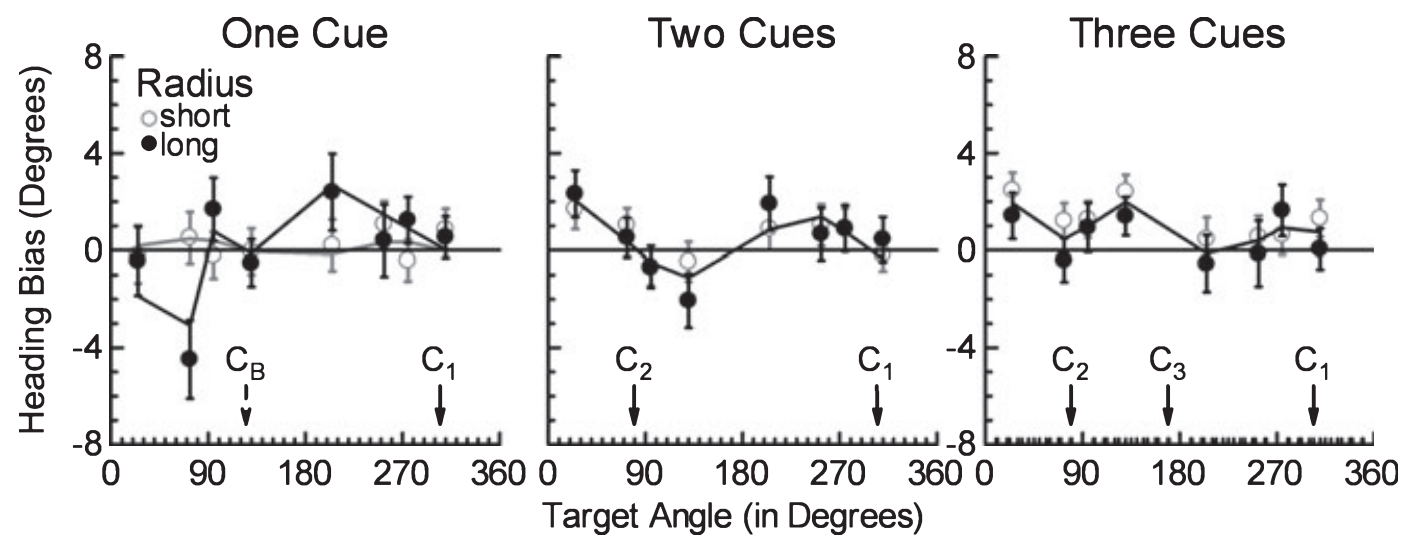

Fig. 6. Fuzzy-boundary model predictions based on Eq. 2a fit to the initial heading bias across target angle separately for the three cue conditions. The error bars indicate 1 standard error of the mean. A positive value indicates a counterclockwise bias, and a negative bias indicates a clockwise bias. $C_{\mathrm{B}}=$ boundary-based cue, $C_{1}=$ cue $1, C_{2}=$ cue 2 , and $C_{3}=$ cue 3 . 
depends on the location of the target. This relationship, however, changes with the number of cues, as indicated by the significant Target Angle $\times$ Cue interaction and the very different shape of the bias patterns shown across the different panels of Fig. 6. The pattern of bias in Fig. 6 suggest a three-way interaction of target angle, cue, and radius, but this effect was not statistically significant (although the Target Angle $\times$ Radius interaction was).

Fig. 6 illustrates the initial heading bias and the flexible fuzzy-boundary model predictions. Based on our flexible fuzzy-boundary model of Eq. 2, we represent the bias in the initial heading as follows:

$$
\mathrm{HB}_{i j}=\lambda \mu_{i j}+(1-\lambda) \sum \mathrm{r}_{i j k} P_{k}-\mu_{i j}
$$

where $\mathrm{HB}_{i j}$ is the initial heading bias for target angle $i$ at radius $j, \mu_{i j}$ is the mean of the distribution of fine-grain memory values for the target $i j$ assumed to be unbiased and hence equated with the true location of the target, and $r_{i j k}$ represents the relevance of prototype $k$ to stimulus $i j$. Similarly, $P_{k}$ is the mean of the distribution of prototype locations for the relevant category. The parameter, $\lambda$, which varies from 0 to 1 , represents the relative weight of the fine-grain information.

The model is an extended version of the category-adjusment model that allows cues to serve as prototypes and assumes probabilistic prototype recruitment. The prototype recruitment equation that describes relevance weights is based on the similarity of the stimulus to the prototype, modeled as follows:

$$
r_{i j k}=\frac{\exp \left(-c_{j k}\left|\mu_{i j}-P_{k}\right|\right)}{\sum \exp \left(-c_{j k}\left|\mu_{i j}-P_{k}\right|\right)},
$$

where $c_{j k}$ is a sensitivity parameter that represents the sharpness of the boundary (the lower the value of $c$, the fuzzier the boundary) and that can vary with radius or prototype. When $c$ does not vary with prototype, the inferred category boundaries fall at equal distances between category prototypes. When $c$ varies with protoype, the inferred category boundary would fall closer to the prototype with the higher value of $c$. For modeling purposes, we include "virtual" prototypes at $360^{\circ}$ above the lowest prototype and $360^{\circ}$ below the highest prototype to account for the fact that $360^{\circ}$ and $0^{\circ}$ represent the same angle and thus provide an accurate recruitment function (for more details, see Fitting, Wedell, et al., 2007). Note that even when there is only one prototype, there will be a boundary opposite that prototype created by the inclusion of these virtual prototypes. Equation $3 \mathrm{~b}$ was substituted for $r_{i j k}$ in Eq. 2a when fitting the data using a least-squared error criterion. In fitting the data, we allowed the number of prototypes to vary and included additional prototypes if they significantly incremented $R^{2}$.

When viewing Fig. 6, inferred prototype locations roughly correspond to where the downward sloping function intersects the $0^{\circ}$ bias axis. As shown, two prototypes were inferred for one-cue and two-cue conditions and three were inferred for the three-cue condition. The fact that bias was close to zero at each of the cue locations supports the idea of a cue-based category structure used to encode spatial locations. As shown in Fig. 6, the model was able to fit the pattern of the data in the one-cue condition well with a five-parameter 
model, including a prototype location close to the actual cue location, a phantom prototype roughly opposite to the cue location and $c$ values varying with radius $(\lambda=0.975$, $c_{\mathrm{LP} 1}=0.312, P_{\mathrm{B}}=125.6^{\circ}, P_{1}=310.9^{\circ}, c_{\mathrm{S}}=0.016, R^{2}=.726$; note that, for the long radius, the sensitivity parameter $c$ was fixed at 1 for the phantom prototype.). Similarly, the best fit model in the two-cue condition was a five-parameter model, with inferred prototypes very close to the cue locations and $c$ varying with prototype $\left(\lambda=0.969, c_{1}=0.026\right.$, $\left.P_{1}=302.5^{\circ}, c_{2}=0.030, P_{2}=84.8^{\circ}, R^{2}=.783\right)$. No significant improvement was noted when including radius or an additional prototype in the model. Finally, the best fit model in the three-cue condition included six parameters, with three prototypes and two values of $c$ that varied with prototype $\left(\lambda=0.919, \quad c_{1}=c_{2}=0.018, \quad P_{1}=34.6^{\circ}, \quad P_{2}=181.2^{\circ}\right.$, $\left.c_{3}=0.013, P_{3}=344.1^{\circ}, R^{2}=.614\right)$. No significant improvement was noted when including radius in the model or additional prototypes in the model. Although the inferred location for $P_{2}$ was very close to a cue value, that for $P_{1}$ was somewhat below the corresponding cue location and that for $P_{3}$ was somewhat above the corresponding cue location. In summary, the flexible-fuzzy boundary gives a moderate account of the cue-based bias pattern by inferring prototypes close to cue locations.

In addition to the significant effects described in Table 3 , the most prominent effect was a main effect of starting location, $F(3,348)=664.64, p<.001$. Fig. 7A illustrates the nature of this bias. As shown, east and west starting locations tended to show an upward bias (reflected in a clockwise bias for the west, $M=-9.65, S D=3.83$, and counterclockwise bias for the east, $M=9.77, S D=3.44$ ). Bias was much less for the north and south locations and tended to reflect a slight leftward bias $\left(M_{\text {north }}=2.94, S D=3.02\right.$, and $M_{\text {south }}=-0.91$, $S D=3.37)$. The impact of starting location varied with orientation, $F(9,1044)=17.77$. As illustrated in Fig. 7B, the east starting location seems to display less initial heading bias for the $270^{\circ}$ orientation compared with the other orientations, whereas the west starting location shows a higher initial heading bias for the $90^{\circ}$ orientation compared with the other orientations. However, the basic pattern of the start location is maintained across all orientations. An additional significant Cue $\times$ Orientation $\times$ Start interaction, $F(18,1044)=9.30$, reflects

(A)

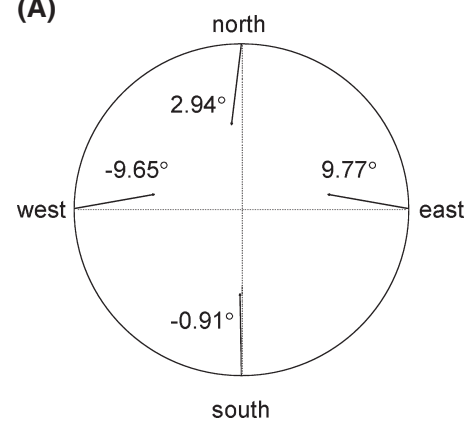

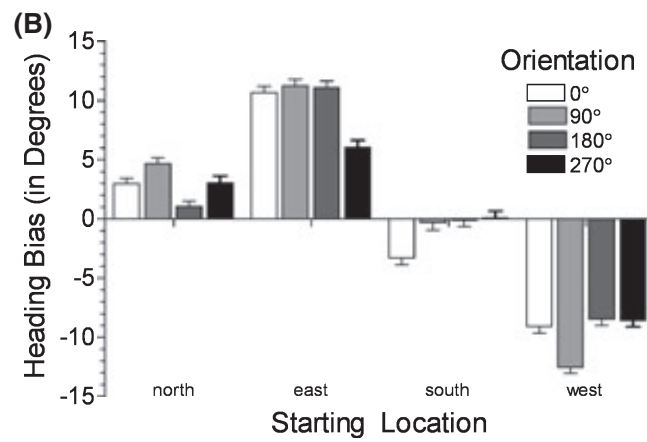

Fig. 7. (A) Circular task field illustrating the significant starting location effect on initial heading bias. (B) Initial heading bias in degrees for the four starting locations separate for orientation. The error bars indicate 1 standard error of the mean. A significant Start $\times$ Orientation interaction was noted. A positive value indicates a counterclockwise bias, and a negative bias indicates a clockwise bias. 
some fluctuation of this pattern with cue, but these effects were not of particular importance to our research focus.

Further significant effects were noted with target angle. A significant Target Angle $\times$ Orientation interaction, $F(21,2436)=14.37, p<.001$, is illustrated in Fig. 8 and reflects a quadrant-based orientation bias. Examining the $0^{\circ}$ rotation condition, one can see a clockwise (negative) bias for quadrant 1 targets, a counterclockwise (positive) bias for quadrant 3 targets, and a mixed pattern for targets in quadrants 2 and 4. In each rotation condition, Fig. 8 codes the targets in terms of the quadrants they end up in after rotation. Note that there is a consistent tendency to maintain the clockwise bias for quadrant 1 locations (white bars) and counterclockwise bias for quadrant 3 locations (dark gray bars). Additional significant effects relative to starting position were found but are not described here as they were small and inconsequential to our hypotheses.

\subsection{End-position bias}

End-position bias was measured by the average location during the last 10 recorded moves before finding the target location. Because this measure only reflects bias effects when collapsed across starting position, we do not include the starting position in the mixed factorial ANOvA. This analysis did include an additional factor, Dimension, that corresponded to the dimension (horizontal or vertical) on which the location was recorded. Consequently, a 3 (Cue) $\times 2$ (Radius) $\times 2$ (Dimension) $\times 8$ (Target Angle) $\times 4$ (Orientation) mixed factorial ANOva was used to analyze the end-position bias, with Table 6 summarizing the key elements of the analysis.

The significant Cue $\times$ Target Angle $\times$ Dimension interaction indicates a significant impact of cue condition on end-position bias for the different target locations. This pattern of result supports $\mathrm{H}_{2 \mathrm{~d}}$, which asserts that end-position bias should be toward the nearest cuebased prototype. To infer prototype locations that may account for this pattern of bias, we fit

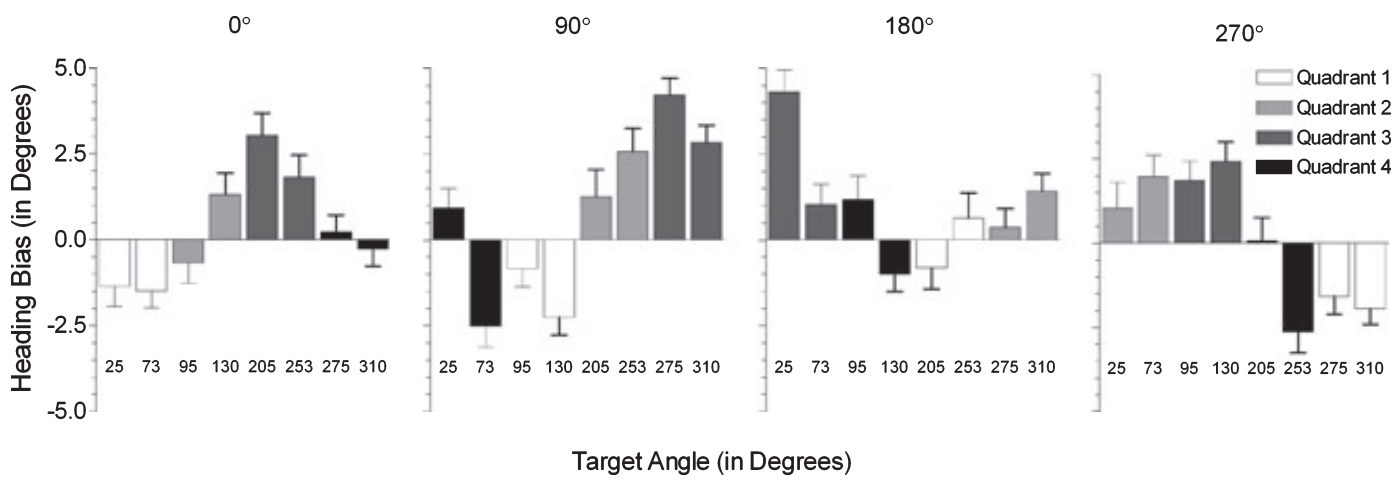

Fig. 8. Initial heading bias in degrees for the four orientations separate for target angle. The error bars indicate 1 standard error of the mean. A significant Target Angle $\times$ Start $\times$ Orientation interaction was noted. A positive value indicates a counterclockwise bias, and a negative bias indicates a clockwise bias. Quadrant refers to the quadrant in which the target was found after the rotation. 
Table 6

Degrees of freedom and $F$-values for 3 (Cue) $\times 2$ (Dimension) $\times 2$ (Radius) $\times 8$ (Target Angle) $\times 4$ (Orientation) $\times 4$ (Start) mixed factorial ANOvAs

\begin{tabular}{lccc}
\hline Source & df & End-Position Bias & Absolute End-Position Error \\
\hline Cue $(C)$ & 2,116 & 1.55 & $14.3^{* * *}$ \\
Radius $(R)$ & 1,116 & 0.10 & 0.32 \\
$R \times C$ & 2,116 & 0.01 & 0.02 \\
Dimension $(D)$ & 1,116 & $7.57^{* *}$ & 0.05 \\
$D \times C$ & 2,116 & $22.20^{* * *}$ & 0.88 \\
$D \times R$ & 1,116 & $5.01^{*}$ & $4.68^{*}$ \\
$D \times C \times R$ & 2,116 & $3.72^{*}$ & 0.05 \\
Target Angle (TA) & 7,812 & 1.26 & $14.99^{* * *}$ \\
TA $\times C$ & 14,812 & 1.00 & $9.83^{* * *}$ \\
TA $\times R$ & 7,812 & 1.09 & $3.97^{* *}$ \\
TA $\times C \times R$ & 14,812 & $1.84^{*}$ & 0.67 \\
$D \times$ TA & 7,812 & $7.51^{* * *}$ & 1.02 \\
$D \times$ TA $\times C$ & 14,812 & $10.96^{* * *}$ & 1.15 \\
$D \times$ TA $\times R$ & 7,812 & 0.88 & 1.29 \\
$D \times$ TA $\times C \times R$ & 14,812 & 0.68 & 1.04 \\
\hline
\end{tabular}

Note: $* p<.05, * * p<.01, * * * p<.001$, for the within-subjects factors: $p_{\mathrm{GG}}$.

a version of the flexible fuzzy-boundary model taken directly from a previous study (Wedell et al., 2007). This basic model is based on Eq. 2, using Cartesian coordinates, and describes the bias in the mean response for stimulus $i$ along dimension $m$ as a weighted average of the actual location on that dimension (assumed to be the mean of the fine-grain memory representation) and the weighted sum of prototype locations along that dimension as follows:

$$
\mathrm{BIAS}_{i j m}=\lambda \mu_{i j m}+(1-\lambda) \sum r_{i j k} P_{k m}-\mu_{i j m},
$$

where Bias $_{i j m}$ is the bias in the mean response for the target corresponding to angle $i$ and radius $j$ on dimension $m$ minus its actual location, $\lambda$ is the relative weight of fine-grain memory, $\mu_{i j m}$ is the mean of the fine-grain memory distribution (corresponding to the actual stimulus location) on dimension $m$ (which takes two values in this case, $x$ and $y$ ), $P_{k m}$ is the location of prototype $k$ on dimension $m$, and $r_{i j k}$ is the relevance weight of prototype $j$ for the target. Note that the fuzzy-boundary notion is captured by intermediate relevance weightings of prototypes so that there may be more than one relevant prototype, especially as the target approaches the border between adjacent categories. In our model, relevance weighting is based on the similarity of the location to the prototype as described in Eq. 3, where similarity is assumed to be a negative exponential function of Euclidean distance:

$$
r_{i j k}=\frac{\exp \left[-c\left(\left(\mu_{i j X}-P_{k X}\right)^{2}+\left(\mu_{i j Y}-P_{k Y}\right)^{2}\right)^{1 / 2}\right]}{\sum \exp \left[-c\left(\left(\mu_{i j X}-P_{k X}\right)^{2}+\left(\mu_{i j Y}-P_{k Y}\right)^{2}\right)^{1 / 2}\right]},
$$

where $c$ is a sensitivity parameter that is fixed in this study at 1 and $r_{i j k}$ indicates the proportionate influence of $P_{k}$ on the estimate of stimulus $i j$, prior to weighting by $(1-\lambda)$. Our 

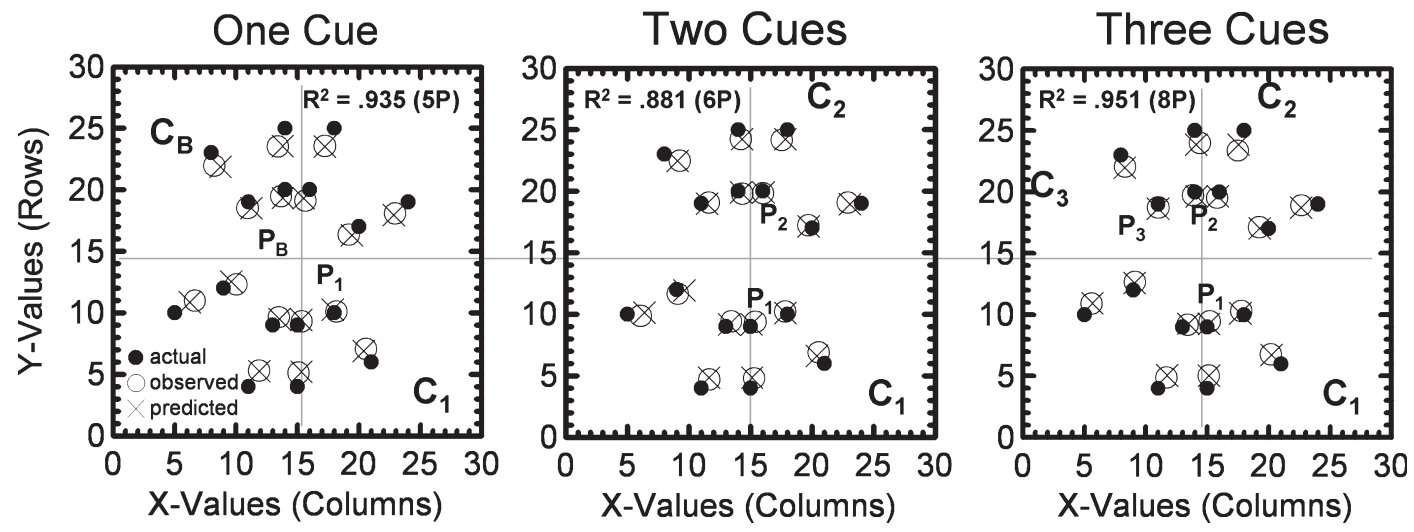

Fig. 9. Model fit based on Eq. $2 \mathrm{~b}$ on the $X$ and $Y$ coordinates of end-position bias separately for the three cue conditions. Open circles illustrate the estimated locations, the solid circles illustrate the actual target locations, and $X \mathrm{~s}$ indicate the model predicted locations. Inferred prototype locations are represented by the symbol $P$. Actual cue locations are represented by the symbol $C$. Subscripts refer to following: $1=$ cue $1, \mathrm{~B}=$ boundary for cue $1,2=$ cue 2 , and $3=$ cue 3 .

modeling strategy was to combine Eqs. $2 \mathrm{~b}$ and $3 \mathrm{c}$ and fit the bias in $x$ and $y$ responses simultaneously after placing different constraints on the number and locations of prototypes. To examine the goodness of fit of the model, we use change in $R^{2}$ as the criterion to determine the best version of the model. To generate the predicted values shown in Fig. 9, we substituted the values from Eq. $2 \mathrm{~b}$ into Eq. 1a:

$$
\mathrm{EST}_{i j m}=\lambda \mu_{i j m}+(1-\lambda) \sum \mathrm{r}_{i j k} P_{k m},
$$

where $\mathrm{EST}_{i j m}$ is the predicted estimate based on the model parameters.

Table 7 presents the parameter values of the best fit models for each cue condition. For the one-cue condition, the model using one prototype and a phantom prototype at the

Table 7

Parameter values and fit indices modeling the $x$ and $y$ coordinates of the end-position bias for each of the three cue conditions

\begin{tabular}{lcccccccccc}
\hline & & \multicolumn{7}{c}{ Parameters } \\
\cline { 3 - 10 } Cue & Model & Radius & $\lambda$ & $P X_{1}$ & $P Y_{1}$ & $P X_{2}$ or $P X_{\mathrm{B}}$ & $P Y_{2}$ or $P X_{\mathrm{B}}$ & $P X_{3}$ & $P Y_{3}$ & $R^{2}$ \\
\hline 1 & $5 \mathrm{P}$ & Short & $\mathbf{0 . 8 4 4}$ & $\mathbf{1 7 . 1}$ & $\mathbf{1 1 . 7}$ & $\mathbf{1 2 . 6}$ & $\mathbf{1 5 . 8}$ & - & - & .936 \\
& & Long & 0.844 & 17.1 & 11.7 & 12.6 & 15.8 & - & - & \\
2 & $6 \mathrm{P}$ & Short & $\mathbf{0 . 9 1 5}$ & $\mathbf{1 6 . 4}$ & $\mathbf{1 8 . 9}$ & $\mathbf{1 5 . 9}$ & $\mathbf{1 1 . 0}$ & - & - & .881 \\
& & Long & $\mathbf{0 . 8 7 7}$ & 16.4 & 18.9 & 15.9 & 11.0 & - & - & \\
3 & \multirow{2}{*}{$8 \mathrm{P}$} & Short & $\mathbf{0 . 8 4 7}$ & $\mathbf{1 5 . 9}$ & $\mathbf{1 0 . 7}$ & $\mathbf{9 . 0}$ & $\mathbf{1 6 . 2}$ & $\mathbf{1 5 . 0}$ & $\mathbf{1 7 . 6}$ & .951 \\
& & Long & $\mathbf{0 . 8 4 5}$ & 15.9 & 10.7 & 9.0 & 16.2 & 15.0 & 17.6 & \\
\hline
\end{tabular}

Note: $\mathrm{P}=$ parameters; $\lambda=$ fine-grain memory; $X$ refers to horizontal value (increasing left to right); $Y$ refers to vertical value (increasing bottom to top); subscripts for $X$ and $Y$ indicate which prototype they refer to; boldfaced values are free to vary; italicized values were constrained to be equal to a bold faced value. 
boundary fit significantly better than a model using only one prototype. Further, using three prototypes did not explain significantly more variance. The same was true for the two-cue condition. For the three-cue condition, a model using one or two prototypes was significantly worse compared with a model using three prototypes. Adding a fourth prototype, however, did not explain significantly more variance.

As shown in Fig. 9, there were systematic bias effects as reflected in the differences between the open symbols (observed values) and filled symbols (actual locations). The model predictions (shown as $X \mathrm{~s}$ ) explained this pattern of bias well, as predictions typically fell very close to observed values. The inferred prototypes from the model are shown in the figure as $P$ s. Note that these prototypes line up very closely with corresponding cue $(C)$ locations (and the boundary location in the one-cue condition), which is clearly consistent with cues being used to determine spatial categories for encoding target locations. The prototypes are shown to be located inside the short radius targets, reflecting to some degree a center-based bias or tendency to assume the target is more central than it really is. These results implicate cue-based bias effects late in the navigational search process. If such effects were not present, then we would expect the average locations over the last 10 moves to be distributed evenly around the targets rather than biased toward cue-based prototypes.

Hypothesis $\mathrm{H}_{1 \mathrm{~d}}$ proposes that the end-position error should be reduced with more cues. To test this, we conducted a parallel analysis on the absolute value of end-position error, corresponding to how far from the target the participant averaged over the last 10 moves. The results are included in the corresponding column of Table 6. The main effect of Cue indicated that the average distance from target varied with the number of cues. The distance was the greatest for one cue $(M=2.05, S D=0.46)$, and less for two cues $(M=1.70$, $S D=0.12)$ and three cues $(M=1.85, S D=0.13)$. Pairwise comparisons using Tukey's correction indicated that the one-cue condition differed significantly from the two-cue condition $(p<.001)$ and from the three-cue condition $(p<.01)$, but the latter two did not differ.

Hypothesis $\mathrm{H}_{3 \mathrm{~d}}$ proposes that the end-position error should be reduced for the $0^{\circ}$ orientation when compared with the other three orientations. The main effect of orientation was marginally significant, $F(3,348)=2.605, p_{\mathrm{GG}}=.056$. A planned contrast comparing the $0^{\circ}$ orientation $(M=1.80, S D=0.30)$ with the other three orientations $\left(M_{90^{\circ}}=1.90\right.$, $S D_{90^{\circ}}=0.05 ; M_{180^{\circ}}=1.88, S D_{180^{\circ}}=0.04 ; M_{270^{\circ}}=1.87, S D_{270^{\circ}}=0.04$ ) did achieve significance, $F(1,121)=6.27, p<.05)$. In addition, there was a significant Orientation $\times$ Cue interaction, $F(6,348)=3.27$. This interaction indicated that orientation had a greater effect in the one-cue condition than in two- or three-cue condition. Thus, these supplemental analyses of absolute error provide support for the hypotheses that, late in the search, cues and orientation still have their hypothesized relations on spatial memory.

\section{Discussion}

In this study, we introduced a new spatial memory task that combined aspects of two well-known spatial tasks: the Morris water maze task and the dot-location task. Our results provide compelling evidence for the robust role that surrounding cues play in spatial 
memory representation, influencing both early and late search behavior. Indeed, the pattern of observed biases and errors were well predicted from models developed from research on the simple dot-location task that utilized a rotating task field (Fitting et al., 2005; Fitting, Wedell, et al., 2007; Fitting et al., 2008a). Because the pattern of results was somewhat complex, we first review the key findings from this experiment.

\subsection{Key findings}

The key findings of the current navigation study can be summarized into three main points. First, memory performance improves with an increase in the number of cues during navigation, supportive of the first main hypothesis. This result is consistent with our previous finding from various memory location tasks (Fitting, Allen, et al., 2007; Fitting, Wedell, et al., 2007; Fitting et al., 2008a, 2008b).

In this study, we found that excess moves, heading error, and absolute end-position error decreased with more cues. In all cases, the one-cue condition had a greater error than the two- and three-cue conditions, which did not differ from one another. Thus, there may be diminishing benefits of extra cues. These are likely to be task dependent, as our human analog of the water maze study showed a significant improvement from two to four cues and our fixed orientation studies only began to show improvements with four or more cues (Fitting, Allen, et al., 2007; Fitting et al., 2008b). Although one might argue that the enhanced memory performance with more cues is a foregone conclusion in a navigation task, other researchers have found essentially no effects when cues are removed on test trials (Jacobs et al., 1998; Nadel et al., 1998). Because we did not vary the number of cues from the learning to the test phase, we cannot say what effect this manipulation would have in the current task. Instead, our results show the importance of cues in stabilizing memory when cues are present at encoding and retrieval. Within the category-adjustment framework, such stabilization reflects a strengthening of fine-grain memory near cues.

Second, and related to this last point, absolute error and bias in remembered locations decreases as a function of proximity to cue locations, supporting the second main hypothesis. For the absolute error measures, this pattern of cue-related error reduction was found in our research using the rotated dot-location task, with absolute error minimized when the target was located close to a cue (Fitting, Wedell, et al., 2007; Fitting et al., 2008a). In this study, it was manifest in the excess moves measures $\left(\mathrm{H}_{2 \mathrm{a}}\right)$ and initial heading error $\left(\mathrm{H}_{2 \mathrm{~b}}\right)$, and it was well described by the proximity-based model of Eq. 4. The convergence of these findings across both tasks supports the idea that cues serve to bolster fine-grain information in proximal regions, with the current navigation task indicating that these effects occur both early and late in the process.

Turning to bias measures, effects were observed early in the search process at the initial heading, manifest in the initial heading bias measure $\left(\mathrm{H}_{2 \mathrm{c}}\right)$ and late in search behavior process during the last 10 moves, manifest in the end-position bias measure $\left(\mathrm{H}_{2 \mathrm{~d}}\right)$. These effects were well predicted by an extension of the previously developed flexible fuzzy-boundary model (Fitting et al., 2005; Fitting, Wedell, et al., 2007; Wedell et al., 2007). Bias measures early and late in the navigational process reflected a shift in remembered locations in the 
direction toward the nearest available external cue. In modeling the initial heading bias, we assumed that cues and targets were encoded in a polar coordinate system as angular vectors from the center of the task field (Fitting et al., 2005; Fitting, Wedell, et al., 2007). As shown in Fig. 6, the pattern of initial heading biases reflected a shift toward the prototype's location, with estimates largely unbiased when targets were aligned with cues (or phantom cues). Not shown in this figure is the overall initial heading bias toward the center of the circle. This bias is also responsible for the reduced initial heading error for short radius targets. Finally, in modeling bias in the last 10 moves, we assumed a Cartesian coordinate system that allowed us to simultaneously model bias in the horizontal and vertical directions (Wedell et al., 2007). As shown in Fig. 9, the angular locations inferred for prototypes were very closely aligned with the corresponding cue locations. The radial locations were well inside the short radius points, suggesting a bias toward the center of the circle late in the search process.

Third, the memory for targets was the best when the task field was presented at the same orientation as in learning, consistent with the basic encoding specificity principle in which memory is the best when cues at retrieval match those at encoding (Tulving \& Thomson, 1973) and summarized in $\mathrm{H}_{3 \mathrm{a}-\mathrm{d}}$. Similar findings were observed in the dot-location task for measures of accuracy, comparing the $0^{\circ}$ rotation trials to trials with rotations of $30^{\circ}, 90^{\circ}$, and $160^{\circ}$ (Fitting, Wedell, et al., 2007; Fitting et al., 2008a). More generally, this finding can also be related to the memory performance in mental rotation tasks, with the literature indicating that mental rotation is associated with an angle-dependent increase in the demands placed on working memory (Harris et al., 2000; Shepard \& Metzler, 1971). Any rotation therefore increases the cognitive cost of encoding, which increases with the degree of rotation, resulting in an increased error and bias. In this study, this hypothesis was supported by the excess moves measure $\left(\mathrm{H}_{3 \mathrm{a}}\right)$, initial heading error $\left(\mathrm{H}_{3 \mathrm{~b}}\right)$, initial heading bias $\left(\mathrm{H}_{3 \mathrm{c}}\right)$, and absolute end-position error $\left(\mathrm{H}_{3 \mathrm{~d}}\right)$. Data in the $0^{\circ}$ rotation condition reflected more accurate spatial estimations compared with data in the $90^{\circ}, 180^{\circ}$, or $270^{\circ}$ rotations.

\subsection{Inferences from the four measures of memory performance}

The current navigation task included four measures. Excess moves and initial heading error index error measures across the entire search and early in search, respectively. Initial heading bias and end-position bias index bias measures early and late in search behavior, respectively. We discuss these measures and their implications for our hypotheses in turn.

\subsubsection{Excess moves}

This measure reflects the overall inaccuracy of place memory and is akin to the typical swimming time measures used in water maze tasks. It is based on the idea that the efficiency in navigation decreases as the memory for spatial target location degrades. Our results for excess moves indicate that the memory for spatial location decreased with a decrease in the number of available cues $\left(\mathrm{H}_{1 \mathrm{a}}\right)$, with distance to the nearest cue $\left(\mathrm{H}_{2 \mathrm{a}}\right)$, and with orientation change of the task field from the presented orientation in the learning trials $\left(\mathrm{H}_{3 \mathrm{a}}\right)$. These effects were well captured by the proximity model, with accuracy decreasing as a function 
of proximity of the target locations to the available external cue. Increasing the number of cues increases the likelihood of a target being close to a cue and hence increases the memory performance, $\mathrm{H}_{1 \mathrm{a}}$. The significant Orientation $\times$ Cue interaction indicated that the effect of cue condition was much larger for the three shifted orientations than for the original $0^{\circ}$ learning orientation, consistent with $\mathrm{H}_{3 \mathrm{a}}$.

\subsubsection{Initial heading error}

This measure reflects the inaccuracy memory representations early in the navigational process. Our results for the initial heading error indicated that, even early in search behavior, the number of cues influenced the navigation process, with the initial heading error reduced when more cues were available $\left(\mathrm{H}_{1 \mathrm{~b}}\right)$. The initial heading error can be interpreted as reflecting both bias and inaccuracy in fine-grain memory. Consistent with $\mathrm{H}_{2 \mathrm{~b}}$ and the excess moves data, the initial heading error was reduced for locations closest to cues. This again supports the idea that cues help to anchor fine-grain memory for locations proximal to them. Given our supposition that fine-grain memory is bolstered near cues, we would have expected the initial heading error to be greater for short radius targets than for long radius targets $\left(\mathrm{H}_{2 \mathrm{~b}}\right)$. However, the opposite result was found, with the initial heading error greater for short radius targets. This finding would appear to contradict our result for excess moves $\left(\mathrm{H}_{2 \mathrm{a}}\right)$ in which short radius targets were harder to find due to reduced fine-grain memory. We reconcile these findings by arguing that the reduction in initial heading error was due to an unanticipated center-bias effect, a tendency to head toward the center of the circle. As short radius targets are closer to the center, this bias offsets poorer fine-grain memory for those targets. The initial heading error was also strongly influenced by the orientation to the task field and the starting location. The effect of orientation effect was demonstrated in excess moves $\left(\mathrm{H}_{3 \mathrm{a}}\right)$. Its occurrence for the initial heading error indicates that the disruptive influence of shifting orientation from the learning orientation occurs early in the navigation process $\left(\mathrm{H}_{3 \mathrm{~b}}\right)$. The starting location effect, on the other hand, was not observed for excess moves and appears to be tied to biases associated with initial heading and discussed below. Overall, the initial heading error measure provided an important perspective on the early effects on spatial accuracy during the course of navigation and might even be considered a measure of a discrimination task rather than a reproduction task. Velea (2006) has recently illustrated the distinction between reproduction and discrimination task by proposing a statistical model that takes a novel approach to the analysis of discrimination responses.

\subsubsection{Initial heading bias}

This measure is expressed using the polar coordinate system and reflects the angular bias effects that occur early in the navigation process. As predicted in $\mathrm{H}_{1 \mathrm{c}}$, an increase in the number of cues revealed a decrease in initial heading bias, due to the fact that bias tends to increase with distance to the nearest cue. Further, as predicted by the flexible fuzzy-boundary model, prototypes were located very close to the cue locations with biases occurring in the direction toward the nearest cue $\left(\mathrm{H}_{2 \mathrm{c}}\right)$. Consistent with past research and with error measures described above, the one-cue condition produced a phantom prototype that was located 
near the boundary opposite to prototype 1 (Fitting et al., 2008a) with the present finding generalizing phantom cue effects to a measure of bias early in navigation. The phantom cue in the one-cue condition might arise from a strategy for locating targets that are quite distant from the single cue, where it would be advantageous to project an imaginary cue at that border and code location relative to it. Note that other researchers have also found evidence for the use of phantom landmarks near a border (Schmidt, Werner, \& Diedrichsen, 2003).

In addition to the hypothesized effects noted above, large effects were noted for starting location and orientation on initial heading bias. The most prominent effect was the effect of starting location, reflecting upward biases for east and west starting locations and a slight leftward bias for north and south starting locations. This basic starting location pattern effect might be due to an overall bias effect in the navigation process that occurs when sitting in front of a two-dimensional computer display. This is supported by recent research (Kelly \& McNamara, 2008) as well as by the fact that even though the impact of starting location is slightly varied by orientation or cue, the main pattern of the starting location effect is maintained, therefore indicating only some small fluctuation. The basic bias linked to orientation was due to a clockwise bias in the first quadrant and a counterclockwise bias in the third quadrant. These biases created Orientation $\times$ Angle effects, as different targets were linked to these quadrants across orientation conditions.

\subsubsection{End-position bias}

This measure reflects the bias effects at the very end of the navigation process and thus late effects of cues on navigation. As hypothesized, prototypes were influenced by the number of available cues, with end-position bias shifting toward the cue-based prototype $\left(\mathrm{H}_{2 \mathrm{~d}}\right)$. This finding was supported by fitting end-position bias data to a basic model directly derived from a previous study (Wedell et al., 2007). Strikingly, the prototype locations derived from the last 10 moves, as shown in Fig. 9, line up closely with the cues and the phantom cue or border location. Predicted bias closely corresponded to the actual bias, reflecting the strength of the model.

To assess where the degree of bias decreased with more cues, we ran supplemental analyses on absolute end-position error. The main effect of the number of cues indicated a decrease in end-position error with more cues, consistent with $\mathrm{H}_{1 \mathrm{~d}}$. The main effect of orientation reflected reduced error in the $0^{\circ}$ orientation, consistent with $\mathrm{H}_{3 \mathrm{~d}}$.

\subsection{Implication for spatial memory representation across different tasks}

The findings of this study indicate that the cue-based spatial memory representations inferred from the dot-location task with rotation generalize to a navigation task within a simple, computer-based environment. The patterns of error and bias from these studies suggest that cues serve two functions. First, they provide the structural categorical representation for remembering spatial locations in an environment with dynamically changing orientation to targets. This conclusion was supported by a systematic bias of estimates toward cue locations that we observe in both paradigms, as well as a systematic tendency to be less biased when close to a cue. Second, cues help anchor fine-grain memory of the spatial 
representation for proximal targets. This conclusion was supported by reductions in error measures for targets closest to the cues. This study indicates that effects on error and bias occur early in the navigation process and persist to the last moves of the navigation process.

The generalization of this cue-based representation across tasks suggests that navigation tasks in real traversable spaces might reflect a similar pattern of observed biases and errors as predicted from models presented in this study as well as in previous works (Fitting et al., 2005; Fitting, Wedell, et al., 2007). For example, these effects might be observed in navigation tasks such as the Morris water maze. In a human analog task of the water maze, we found evidence for cue-based bias in the memory for spatial location (Fitting, Allen, et al., 2007). However, that task examined only end-point bias, used only three targets, and occurred in a vista space. Nevertheless, it suggests that these results might generalize to tasks using a first-person perspective to navigate in a larger environment. An important avenue for future research will be to test how categorical and fine-grain effects of cues may apply to real and virtual environments, when navigation through a vista and large-scale environment is required. Navigation literature incorporates a distinction between high-fidelity and coarser information used in egocentric or allocentric orientations (Mou, McNamara, Valiquette, \& Rump, 2004; Wang \& Spelke, 2000). The two-level approach to spatial memory appears to be well established across a number of different spatial memory paradigms. Although much navigation work in virtual-reality environments has been conducted, these studies have not examined the representational hypotheses we examined in this current study (e.g., Jacobs et al., 1997; Skelton et al., 2006; Waller \& Lippa, 2007). Further, although we believe that the current study provides strong support for the flexible fuzzy-boundary model, other models of geometric category biases may be worth investigating in this context, such as the dynamic field theory of spatial working memory (Spencer, Simmering, \& Schutte, 2006).

Additionally, it might be interesting to investigate the underlying process of spatial memory in map use. A recent study examined whether cues would be used in the categorical coding process within a static environment, with findings indicating that cues were largely ignored in determining the categorical structure of spatial coding and supporting the maintenance of a cue-independent category structure based on geometrically fixed categories rather than cue-based categories (Fitting et al., 2008b). Despite a lack of cue effects on the categorization scheme, findings, however, provided strong evidence that error and inconsistency of estimation were reduced in the cue conditions, thus indicating some impact of cues. Such results have important implications when assessing memory processes in map users or map designers.

When viewing a map from a fixed orientation, it is possible that one would ignore a cue-based representation (i.e., by landmarks) and develop geometric-based spatial coding (i.e., by cardinal directions). However, our studies indicate that when navigating a space in which orientation varies, the cue-based orientation is preferred. Thus, we would predict important consequences of map encoding based on orientation. Our approach provides a quantitative method to assess biases and errors and infer the underlying spatial representation. 


\section{Notes}

1. Participants were able to continuously move the mouse image within the task field, but the image would typically jitter rather than move smoothly in a given direction. For example, if the participant moved the mouse on a $30^{\circ}$ angle, the image would move through the closest adjacent square and continue doing so, jiggling up or down as the movement proceeded in the desired direction. Participants got used to moving the mouse image in the preliminary practice trials and did not indicate frustration with the jittering image.

2. We tried several different approaches for predicting excess moves and initial heading error as a function of distance to cues. All of these did a fairly good job of predicting cue-based relationships. We selected the model described here because it is consistent with our theoretical framework and produced high $R^{2}$ values.

3. The only other significant effect for excess moves was a three-way interaction of target angle, orientation, and starting position, $F(63,7308)=1.83, p<.01$. Because this effect was small and effects related to the starting position are not of particular relevance to the current set of hypotheses, we do not consider this effect further.

4. For the interested reader, the effects of initial heading error for orientation were as follows: Orientation: $F(3,348)=14.41, p<.001$; Target Angle $\times$ Orientation: $F(21$, $2436)=28.75, p<.001$; Radius $\times$ Target Angle $\times$ Orientation: $F(21,2436)=3.03$, $p<.001$; Orientation $\times$ Start: $F(9,1044)=7.45, p<.001 ;$ Cue $\times$ Orientation $\times$ Start: $F(18,1044)=3.29, p<.001 ;$ Target Angle $\times$ Orientation $\times$ Start: $F(63,7308)=$ $12.07, p<.001$; Radius $\times$ Target Angle $\times$ Orientation $\times$ Start: $F(63,7308)=2.41$, $p<.001$. The significant effects for starting location were as follows: Start: $F(3$, $348)=84.21, p<.001 ;$ Cue $\times$ Target Angle $\times$ Start: $F(42,2436)=2.05, p<.001$; and four were significant with including orientation (see above).

\section{References}

Astur, R. S., Ortiz, M. L., \& Sutherland, R. J. (1998). A characterization of performance by men and women in a virtual Morris water task: A large and reliable sex difference. Behavioural Brain Research, 93, 185-190.

Astur, R. S., Taylor, L. B., Mamelak, A. N., Philpott, L., \& Sutherland, R. J. (2002). Humans with hippocampus damage display severe spatial memory impairments in a virtual Morris water task. Behavioural Brain Research, 132, 77-84.

Crawford, L. E., Margolies, S. M., Drake, J. T., \& Murphy, M. E. (2006). Affect biases memory of location: Evidence for the spatial representation of affect. Cognition \& Emotion, 20, 1153-1169.

Fitting, S., Allen, G. L., \& Wedell, D. H. (2007). Remembering places in space: A human analog study of the Morris water maze. In Th. Barkowsky, M. Knauff, G. Ligozat, \& D. R. Montello (Eds.), Spatial cognition V, LNAI 4387 (pp. 59-75). Berlin: Springer.

Fitting, S., Wedell, D. H., \& Allen, G. L. (2005). Memory for spatial location: Influences of environmental cues and task field rotation. In A. G. Cohn \& D. M. Mark (Eds.), COSIT 2005, LNCS 3693 (pp. 459-474). Berlin: Springer-Verlag. 
Fitting, S., Wedell, D. H., \& Allen, G. L. (2007). Memory for spatial location: Cue effects as a function of field rotation. Memory \& Cognition, 35, 1641-1658.

Fitting, S., Wedell, D. H., \& Allen, G. L. (2008a). External cue effects on memory for spatial location within a rotated task field. Spatial Cognition \& Computation, 8, 219-251.

Fitting, S., Wedell, D. H., \& Allen, G. L. (2008b). Cue usage in memory for location when orientation is fixed. Memory \& Cognition, 36, 1196-1216.

Greenhouse, S. W., \& Geisser, S. (1959). On methods in the analysis of profile data. Psychometrika, 32, 95-112.

Grön, G., Wunderlich, A. P., Spitzer, M., Tomczak, R., \& Riepe, M. W. (2000). Brain activation during human navigation: Gender-different neural networks as substrate of performance. Nature Neuroscience, 3, 404-408.

Harris, I. M., Egan, G. F., Sonkkila, C., Tochon-Danguy, H. J., Paxinos, G., \& Watson, J. D. G. (2000). Selective right parietal lobe activation during mental rotation. Brain, 123, 65-73.

Hund, A. M., \& Plumert, J. M. (2002). Delay-induced bias in children's memory for location. Child Development, 73(3), 829-840.

Hund, A. M., \& Plumert, J. M. (2003). Does information about what things are influence children's memory for where things are? Developmental Psychology, 39, 939-948.

Hund, A. M., \& Plumert, J. M. (2005). The stability and flexibility of spatial categories. Cognitive Psychology, $50,1-44$.

Huttenlocher, J., Hedges, L. V., Corrigan, B., \& Crawford, L. E. (2004). Spatial categories and the estimation of location. Cognition, 93, 75-97.

Huttenlocher, J., Hedges, L. V., \& Duncan, S. (1991). Categories and particulars: Prototype effects in estimating spatial location. Psychological Review, 98, 352-376.

Huttenlocher, J., Hedges, L. V., Lourenco, S. F., Crawford, L. E., \& Corrigan, B. (2007). Estimating stimuli from contrasting categories: Truncation due to boundaries. Journal of Experimental Psychology: General, $136,502-519$.

Jacobs, W. J., Laurance, H. E., \& Thomas, K. G. F. (1997). Place learning in virtual space I: Acquisition, overshadowing, and transfer. Learning and Motivation, 28, 521-541.

Jacobs, W. J., Thomas, K. G. F., Laurance, H. E., \& Nadel, L. (1998). Place learning in virtual space II. Topographical relations as one dimension of stimulus control. Learning \& Motivation, 29, 288-308.

Jarrard, L. E. (1993). On the role of the hippocampus in learning and memory in the rat. Behavioral \& Neural Biology, 60, 9-26.

Kelly, J. W., \& McNamara, T. P. (2008). Spatial memories of virtual environments: How egocentric experience, intrinsic structure, and extrinsic structure interact. Psychonomic Bulletin \& Review, 15, 322-327.

Maguire, E. A., Frith, C. D., Burgess, N., Donnett, J. G., \& O'Keefe, J. (1998). Knowing where things are: Parahippocampal involvement in encoding object locations in virtual large-scale space. Journal of Cognitive Neuroscience, 10, 61-76.

Moffat, S. D., \& Resnick, S. M. (2002). Effects of age on virtual environment place navigation and allocentric cognitive mapping. Behavioral Neuroscience, 116, 851-859.

Morris, R. G. M. (1981). Spatial localization does not require the presence of local cues. Learning \& Motivation, 12, 239-260.

Morris, R. G. M., Garrud, P., Rawlins, J. N. P., \& O’Keefe, J. (1982). Place navigation impaired in rats with hippocampal lesions. Nature, 297, 681-683.

Morris, R. G., \& Parslow, D. M. (2004). Neurocognitive components of spatial memory. In G. Allen (Ed.), Human spatial memory: Remembering where (pp. 217-247). Mahwah, NJ: Lawrence Erlbaum Associates.

Mou, W., McNamara, T. P., Valiquette, C. M., \& Rump, B. (2004). Allocentric and egocentric updating of spatial memories. Journal of Experimental Psychology: Learning, Memory, \& Cognition, 30, 142-157.

Nadel, L. (1990). Varieties of spatial cognition: Psychobiological considerations. In A. Diamond (Ed.), Annals of the New York Academy of Sciences (Vol. 608; pp. 613-636). New York: New York Academy of Sciences.

Nadel, L., Thomas, K. G. F., Laurance, H. E., Skelton, R., Tal, T., \& Jacobs, W. J. (1998). Human place learning in a computer generated arena. In C. Freksa, C. Habel, \& K. F. Wender (Eds.), Spatial cognition: 
An interdisciplinary approach to representing and processing spatial knowledge (pp. 399-427). Berlin: Springer.

Newcombe, N. S., \& Huttenlocher, J. (2000). Making space: The development of spatial representation and reasoning. Cambridge, MA: MIT Press.

O'Keefe, J., \& Nadel, L. (1978). The hippocampus as a cognitive map. Oxford, England: Clarendon Press.

Parslow, D. M., Morris, R. G., Fleminger, S., Rahman, O., Abrahams, S., \& Recce, M. (2005). Allocentric spatal memory in humans with hippocampal lesions. Acta Psychologica, 118, 123-147.

Prusky, G. T., West, P. W. R., \& Douglas, R. M. (2000). Reduced visual acuity impairs place but not cued learning in the Morris water task. Behavioural Brain Research, 116, 135-140.

Sandstrom, N., Kaufman, J., \& Huettel, S. A. (1998). Males and females use different distal cues in a virtual environment navigation task. Cognitive Brain Research, 6, 351-360.

Schmidt, T., Werner, S., \& Diedrichsen, J. (2003). Spatial distortions induced by multiple visual landmarks: How local distortions combine to produce complex distortion patterns. Perception \& Psychophysics, 65, 861-873.

Shepard, R. N., \& Metzler, J. (1971). Mental rotation of three-dimensional objects. Science, 171(972), 701-703.

Skelton, R. W., Bukach, C. M., Laurance, H. E., Thomas, K. G. F., \& Jacobs, W. J. (2000). Humans with traumatic brain injuries show place-learning deficits in computer-generated virtual space. Journal of Clinical and Experimental Neuropsychology, 22, 157-175.

Skelton, R. W., Ross, S. P., Nerad, L., \& Livingstone, S. A. (2006). Human spatial navigation deficits after traumatic brain injury shown in the arena maze, a virtual Morris water maze. Brain Injury, 20, 189-203.

Spencer, J. P., \& Hund, A. A. (2002). Prototypes and particulars: Geometric and experience-dependent spatial categories. Journal of Experimental Psychology: General, 131, 16-37.

Spencer, J. P., Simmering, V. R., \& Schutte, A. R. (2006). Toward a formal theory of flexible spatial behavior: Geometric category biases generalize across pointing and verbal response types. Journal of Experimental Psychology: Human Perception \& Performance, 32, 473-490.

Sutherland, R. J., \& Rudy, J. W. (1987). Configural association theory: The role of the hippocampal formation in learning, memory, and amnesia. Psychobiology, 17, 129-144.

Tulving, E., \& Thomson, D. M. (1973). Encoding specificity and retrieval processes in episodic memory. Psychological Review, 80, 352-373.

Velea, J. L. (2006). Recovering stimuli from memory: A statistical method for linking discrimination and reproduction responses. British Journal of Mathematical and Statistical Psychology, 59, 321-346.

Waller, D., \& Lippa, Y. (2007). Landmarks as beacons and associative cues: Their role in route learning. Memory \& Cognition, 35, 910-924.

Wang, R. F., \& Spelke, E. S. (2000). Updating egocentric representations in human navigation. Cognition, 77, 215-250.

Wedell, D. H., Fitting, S., \& Allen, G. L. (2007). Shape effects on memory for location. Psychonomic Bulletin \& Review, 14, 681-686.

Werner, S. \& Diedrichsen, J. (2002). The time course of spatial memory distortions. Memory \& Cognition, $30,718-730$. 